\title{
A lower bound on the spectrum of the sublaplacian
}

\author{
Amine Aribi, Sorin Dragomir ${ }^{1}$, Ahmad El Soufi ${ }^{2}$
}

\begin{abstract}
Aвstract. We establish a new lower bound on the first nonzero eigenvalue $\lambda_{1}(\theta)$ of the sublaplacian $\Delta_{b}$ on a compact strictly pseudoconvex CR manifold $M$ carrying a contact form $\theta$ whose Tanaka-Webster connection has pseudohermitian Ricci curvature bounded from below.
\end{abstract}

\section{INTRODUCTION AND STATEMENT OF MAIN RESULT}

By a classical result of A. Lichnerowicz (cf. Theorem D.I.1 in [8], p. 179) and M. Obata (cf. [34]) for any $m$-dimensional compact Riemannian manifold $(M, g)$ with $\operatorname{Ric}_{g} \geq k g$ the first nonzero eigenvalue $\lambda_{1}(g)$ of the Laplace-Beltrami operator $\Delta_{g}$ satisfies the estimate

$$
\lambda_{1}(g) \geq m k /(m-1)
$$

with equality if and only if $M$ is isometric to the unit sphere $S^{m} \subset \mathbb{R}^{m+1}$. The main ingredient in the proof of (1) is the Bochner-Lichnerowicz formula (cf. e.g. (G.IV.5) in [8], p. 131)

$$
-\frac{1}{2} \Delta_{g}\left(\|d u\|^{2}\right)=\|\operatorname{Hess}(u)\|^{2}-g\left(D u, D \Delta_{g} u\right)+\operatorname{Ric}_{g}(D u, D u)
$$

for any $u \in C^{\infty}(M, \mathbb{R})$. The great fascination exerted by the LichnerowiczObata theorem on the mathematical community in the last fifty years prompted the many attempts to extend (2) and (1) to other geometric contexts e.g. to Riemannian foliation theory (cf. S-D. Jung \& K-R. Lee \& K. Richardson, [27], J. Lee \& K. Richardson, [31], H-K. Pak \& J-H. Park, [36]), to $\mathrm{CR}$ and pseudohermitian geometry (cf. E. Barletta \& S. Dragomir, [3], E. Barletta, [4], S-C. Chang \& H-L. Chiu, [10], H-L. Chiu, [11], A. Greenleaf, [23], S. Ivanov \& D. Vassilev, [24], S-Y. Li \& H-S. Luk, [32]) and to sub-Riemannian geometry (cf. F. Baudoin \& N. Garofalo, [7]). The present paper is devoted to a version of the estimate (1) occurring in CR geometry. Given a compact strictly pseudoconvex CR manifold $\left(M, T_{1,0}(M)\right)$ endowed with a positively oriented contact form $\theta$, the pseudohermitian manifold

\footnotetext{
${ }^{1}$ Dipartimento di Matematica, Informatica ed Economia, Università degli Studi della Basilicata, Viale dell' Ateneo Lucano 10, Campus Macchia Romana, 85100 Potenza, Italy, sorin.dragomir@unibas.it

${ }^{2}$ Laboratoire de Mathématiques et Physique Théorique, Université François Rabelais, Tours, France, amine. aribi@lmpt . univ-tours. fr, Ahmad.Elsoufi@lmpt . univ-tours . fr
} 
$(M, \theta)$ carries a natural second order, positive, formally self-adjoint operator $\Delta_{b}$ (the sublaplacian of $(M, \theta)$ ), formally similar to the Laplacian in Riemannian geometry, yet only degenerate elliptic (in the sense of J-M. Bony, [9]). However $\Delta_{b}$ is hypoelliptic and (by a result of A. Menikoff \& J. Sjöstrand, [33]) it has a discrete spectrum

$$
\begin{gathered}
\sigma\left(\Delta_{b}\right)=\left\{\lambda_{v}(\theta): v \in \mathbb{Z}, \quad v \geq 0\right\}, \quad \lim _{v \rightarrow \infty} \lambda_{v}=+\infty, \\
\lambda_{0}(\theta)=0, \quad \lambda_{v}(\theta) \leq \lambda_{v+1}(\theta), \quad v \geq 1 .
\end{gathered}
$$

On the other hand, by a result of N.Tanaka, [37], and S.M. Webster, [38], $(M, \theta)$ carries a natural linear connection $\nabla$ (the Tanaka-Webster connection of $(M, \theta)$, cf. also [12], p. 25) whose Ricci tensor field is formally similar to Ricci curvature in Riemannian geometry. It is then a natural problem to look for a lower bound on $\lambda_{1}(\theta)$ whenever $\operatorname{Ric}_{\nabla}$ is bounded from below. By strict pseudoconvexity $(M, \theta)$ also carries a natural Riemannian metric $g_{\theta}$ (the Webster metric of $(M, \theta)$, cf. [12], p. 9) whose associated Riemannian volume form is (up a multiplicative constant depending only on the CR dimension $n) \Psi_{\theta}=\theta \wedge(d \theta)^{n}$. Let $\operatorname{div}: \mathfrak{X}(M) \rightarrow C^{\infty}(M, \mathbb{R})$ be the divergence operator associated to the volume form $\Psi_{\theta}$. Then the sublaplacian may be written in divergence form as $\Delta_{b} u=-\operatorname{div}\left(\nabla^{H} u\right)$ where $\nabla^{H} u$ (the horizontal gradient) is the projection of the gradient $\nabla u$ with respect to $g_{\theta}$, on the Levi, or maximally complex, distribution $H(M)=\operatorname{Re}\left\{T_{1,0}(M) \oplus T_{0,1}(M)\right\}$. Consequently the horizontal gradient $\nabla^{H} u$ is the pseudohermitian analog to the gradient $D u$ in Riemannian geometry. The first step is then to produce a pseudohermitian version of (2) i.e. compute $\Delta_{b}\left(\left\|\nabla^{H} u\right\|^{2}\right)$ (for an arbitrary eigenfunction $u$ of $\Delta_{b}$ ) in terms of the pseudohermitian Hessian $\nabla^{2} u$ and the Ricci curvature Ric $_{\nabla}$ of the Tanaka-Webster connection. The first to realize the difficulties in producing a pseudohermitian analog to (2) was A. Greenleaf, [23]. Indeed his Bochner-Lichnerowicz type formula

(3) $\frac{1}{2} \Delta_{b}\left(\left\|\nabla^{1,0} u\right\|^{2}\right)=\sum_{\alpha, \beta}\left(u_{\alpha \bar{\beta}} u_{\bar{\alpha} \beta}+u_{\alpha \beta} u_{\bar{\alpha} \bar{\beta}}\right)+2 i \sum_{\alpha}\left(u_{\bar{\alpha}} u_{0 \alpha}-u_{\alpha} u_{0 \bar{\alpha}}\right)+$

$+\sum_{\alpha, \beta} R_{\alpha \bar{\beta}} u_{\bar{\alpha}} u_{\beta}+i n \sum_{\alpha, \beta}\left(A_{\alpha \beta} u_{\bar{\alpha}} u_{\bar{\beta}}-A_{\bar{\alpha} \bar{\beta}} u_{\alpha} u_{\beta}\right)+\frac{1}{2} \sum_{\alpha}\left\{u_{\bar{\alpha}}\left(\Delta_{b} u\right)_{\alpha}+u_{\alpha}\left(\Delta_{b} u\right)_{\bar{\alpha}}\right\}$

involves the torsion terms $A_{\alpha \beta}$ (possessing no Riemannian counterpart). Here $\nabla^{1,0} u=\sum_{\alpha} u_{\bar{\alpha}} T_{\alpha}$ (notations and conventions as used in (3) are explained in $\S 2)$. However the attempt to confine oneself to the class of Sasakian manifolds $\left(M, g_{\theta}\right)$ (as in [4], since Sasakian metrics $g_{\theta}$ have vanishing pseudohermitian torsion i.e. $A_{\alpha \beta}=0$ ) isn't successful either: while torsion terms may actually be controlled (when exploiting (3) integrated over $M)$ by the $L^{2}$ norm of $\nabla^{H} u$, the main technical difficulties really arise from the occurrence of terms $\sum_{\alpha}\left(u_{\bar{\alpha}} u_{0 \alpha}-u_{\alpha} u_{0 \bar{\alpha}}\right)$ containing covariant derivatives 
of $\nabla^{H} u$ in the "bad" real direction $T$ transverse to $H(M)$ (the Reeb vector field of $(M, \theta))$.

The novelty brought by the present paper is to establish first a version of Bochner-Lichnerowicz formula for a natural Lorentzian metric $F_{\theta}$ (the Fefferman metric of $(M, \theta)$, cf. [29], [21]) on the total space of the canonical circle bundle $S^{1} \rightarrow C(M) \stackrel{\pi}{\longrightarrow} M$. Fefferman metric $F_{\theta}$ was discovered by C. Fefferman, [20], in connection with the study of boundary behavior of the Bergman kernel of a strictly pseudoconvex domain in $\mathbb{C}^{n}$. An array of problems of major interest in CR geometry e.g. the CR Yamabe problem, [25], the study of subelliptic harmonic maps, [26], and YangMills fields on CR manifolds, [6], are closely tied to the geometry of the Lorentzian manifold $\left(C(M), F_{\theta}\right)$. Indeed the aforementioned problems are projections on $M$ via $\pi: C(M) \rightarrow M$ of Lorentzian analogs to the corresponding Riemannian problems, as prompted by J.M. Lee's discovery (cf. [29]) that $\pi_{*} \square=\Delta_{b}$, where $\square$ is the Laplace-Beltrami operator of $F_{\theta}$ (the wave operator on $\left(C(M), F_{\theta}\right)$ ). For instance any $S^{1}$-invariant harmonic map $\Phi:\left(C(M), F_{\theta}\right) \rightarrow N$ into a Riemannian manifold $N$ projects on a subelliptic harmonic map $\phi: M \rightarrow N$ (in the sense of [26] and [6]). The arguments in [8] carry over in a straightforward manner (cf. our §3) to Lorenzian geometry and give (cf. (21) below)

(4) $-\frac{1}{2} \square\left(F_{\theta}(D f, D f)\right)=F_{\theta}^{*}\left(D^{2} f, D^{2} f\right)-(D f)(\square f)+\operatorname{Ric}_{D}(D f, D f)$

and the corresponding integral formula (22). The projection on $M$ of (4) then leads to another analog (similar to A. Greenleaf's formula (3)) to Bochner-Lichnerowicz formula and then to a new lower bound on $\lambda_{1}(\theta)$. Precisely we may state

Theorem 1. Let $M$ be a compact, strictly pseudoconvex, CR manifold of $C R$ dimension $n$. Let $\theta \in \mathcal{P}_{+}$be a positively oriented contact form on $M$ and $\Delta_{b}$ the corresponding sublaplacian. Let $\operatorname{Ric}_{\nabla}$ be the Ricci tensor of the Tanaka-Webster connection $\nabla$ of $(M, \theta)$ and $\lambda_{1}(\theta) \in \sigma\left(\Delta_{b}\right)$ the first nonzero eigenvalue of $\Delta_{b}$. If

$$
\operatorname{Ric}_{\nabla}(X, X) \geq k G_{\theta}(X, X)
$$

for some constant $k>0$ and any $X \in H(M)$ then

$$
\lambda_{1}(\theta) \geq \frac{2 n}{(n+2)(n+3)}\left\{(n+3) k-(11 n+19) \tau_{0}-\frac{\rho_{0}}{2(n+1)}\right\}
$$

with $\tau_{0}=\sup _{x \in M}\|\tau\|_{x}$ and $\rho_{0}=\sup _{x \in M} \rho(x) \geq n k$, where $\tau$ and $\rho$ are respectively the pseudohermitian torsion and scalar curvature of $(M, \theta)$.

The lower bound (6) is nontrivial only for $k$ sufficiently large (i.e. $k$ must satisfy (89) in $\S 6$ ). Let $\left(M, g_{\theta}\right)$ be a Sasakian manifold (equivalently $\tau=0$, 
cf. e.g. [12]). Then under the same assumption (i.e. (5) in Theorem 1) A. Greenleaf established the estimate (cf. [23])

$$
\lambda_{1}(\theta) \geq \frac{n k}{n+1} .
$$

Lower bound (6) is sharper that (7) when

$$
k>\frac{\rho_{0}}{n(n+3)} \text {. }
$$

If for instance $M=S^{2 n+1}$ is the standard sphere in $\mathbb{C}^{n+1}$, endowed with the canonical contact form $\theta=(i / 2)(\bar{\partial}-\partial)|z|^{2}$, then $\rho_{0}=2 n(n+1)$ and $k=2(n+1)$ hence (8) holds (and (6) is sharper than (7)).

The essentials of CR and pseudohermitian geometry are recalled in $\S 2$ (by following mainly [12]). The projection of (4) on $M$ gives

$$
\begin{gathered}
-\frac{1}{2} \Delta_{b}\left(\left\|\nabla^{H} u\right\|^{2}\right)=\left\|\Pi_{H} \nabla^{2} u\right\|^{2}-\left(\nabla^{H} u\right)\left(\Delta_{b} u\right)+ \\
+4\left(J \nabla^{H} u\right)\left(u_{0}\right)-\frac{3(n+1)}{n+2} A\left(\nabla^{H} u, J \nabla^{H} u\right)+ \\
+\frac{n+3}{n+2} \operatorname{Ric}_{\nabla}\left(\nabla^{H} u, \nabla^{H} u\right)-\frac{\rho}{2(n+1)(n+2)}\left\|\nabla^{H} u\right\|^{2}
\end{gathered}
$$

(the pseudohermitian Bochner-Lichnerowicz formula, cf. (79) in §5) and the corresponding integral formula (80). The main technical difficulty in the derivation of (9) is to compute the Ricci curvature $\mathrm{Ric}_{D}$ of the Lorentzian manifold $\left(C(M), F_{\theta}\right)$. This is performed by relating the Levi-Civita connection $D$ of $\left(C(M), F_{\theta}\right)$ to the Tanaka-Webster connection $\nabla$ of $(M, \theta)$ (cf. (23)-(27) in $\S 4$, a result got in [6]) and adapting to $S^{1} \rightarrow C(M) \rightarrow M$ a technique originating in the theory of Riemannian submersions (cf. [35]) and shown to work in spite of the fact that $\pi:\left(C(M), F_{\theta}\right) \rightarrow\left(M, g_{\theta}\right)$ isn't a semi-Riemannian submersion (fibres of $\pi$ are degenerate). The relationship among $D$ and $\nabla$ may then be exploited to compute the full curvature tensor $R^{D}$. Only its trace Ric $_{D}$ is evaluated in [29] and the formula there appears as too involved to be of practical use. Our result (cf. (50)-(54) in Lemma 3 below) is simple, elegant and local frame free. This springs from the decompositions $T(C(M))=\operatorname{Ker}(\sigma) \oplus \mathbb{R} S$ and $\operatorname{Ker}(\sigma)=H(M)^{\uparrow} \oplus \mathbb{R} T^{\uparrow}$, themselves relying on the discovery (due to C.R. Graham, [21]) that $\sigma \in \Omega^{1}(C(M)$ ) (given by (20) below) is a connection 1-form in the principal circle bundle $S^{1} \rightarrow C(M) \rightarrow M$. As a byproduct of Lemma 3 one reobtains the result by J.M. Lee, [29], that none of the Fefferman metrics $\left\{F_{\theta} \in \operatorname{Lor}(C(M)): \theta \in\right.$ $\left.\mathcal{P}_{+}\right\}$is Einstein. Integration of (9) over $M$ produces (by (78)) terms $\left\|u_{0}\right\|_{L^{2}}$ where $u_{0} \equiv T(u)$ and $u$ is an arbitrary eigenfunction of $\Delta_{b}$, corresponding to a fixed eigenvalue $\lambda \in \sigma\left(\Delta_{b}\right)$. The $L^{2}$ norm of the (restriction to the Levi distribution $H(M)$ of the) pseudohermitian Hessian $\Pi_{H} \nabla^{2} u$ is estimated by 
using (82) (a result got in [4]). Torsion terms and Ricci curvature terms are respectively estimated by (87) and as a consequence of the assumption (5) in Theorem 1 (together with (86)). Finally to control $\left\|u_{0}\right\|_{L^{2}}$ one exploits a fundamental result got in [10], and referred hereafter as the Chang-Chiu inequality (cf. (91) in Appendix A).

Spectral geometry (spectrae of Laplace-Beltrami and Schrödinger operators) on compact Riemannian manifolds has been intensely investigated over the last twenty-five years by A. El Soufi \& S. Ilias, [13], A. El Soufi \& S. Ilias \& A. Ros, [14], A. El Soufi \& B. Colbois, [15], A. El Soufi \& B. Colbois \& E. Dryden, [16], A. El Soufi \& N. Moukadem, [17], A. El Soufi \& H. Giacomini \& M. Jazar, [18], and A. El Soufi \& R. Kiwan, [19]. A program aiming to recovering the quoted works in the realm of $\mathrm{CR}$ and pseudohermitian geometry was recently started by A. Aribi \& A. El Soufi, [1]. As part of that program A. Aribi \& S. Dragomir \& A. El Soufi studied (cf. [2]) the dependence of spectrae of sublaplacians on the given contact form. The present work is another step on this path (studying spectrae of compact strictly pseudoconvex CR manifolds).

The paper is organized as follows. In $\S 2$ we recall the needed elements of calculus on a pseudohermitian manifold (including the curvature theory for the Tanaka-Webster conection, cf. [37], [38] and [12]). The Lorentzian analog (4) to the Bochner-Lichnerowicz formula (2) is derived in $\S 3$. The technicalities on curvature theory (needed to project (4) on $M$ ) are dealt with in $\S 4$. In $\S 5$ we relate the Lorentzian Hessian $D^{2}(u \circ \pi)$ to the pseudohermitian Hessian $\nabla^{2} u$ and derive the pseudohermitian Bochner-Lichnerowicz formula (9). The lower bound (1) is proved in $\S 6$. Appendix A contains a proof of the Chang-Chiu inequality.

\section{A REMINDER OF CR GEOMETRY}

For all definitions and basic conventions in $\mathrm{CR}$ and pseudohermitian geometry we rely on [12]. Let $\left(M, T_{1,0}(M)\right)$ be an orientable CR manifold, of CR dimension $n$, and let $\bar{\partial}_{b}$ be the tangential Cauchy-Riemann operator. A CR function is a $C^{1}$ solution to the tangential C-R equations $\bar{\partial}_{b} f=0$. Let $H(M)$ be the maximally complex, or Levi, distribution on $M$ and let $J$ be its complex structure. Let $\mathcal{P}$ be the space of all pseudohermitian structures on $M$. For each $\theta \in \mathcal{P}$ the Levi form is $G_{\theta}(X, Y)=(d \theta)(X, J Y)$ for every $X, Y \in H(M)$. If $M$ is nondegenerate then each $\theta \in \mathcal{P}$ is a contact form i.e. $\Psi_{\theta}=\theta \wedge(d \theta)^{n}$ is a volume form on $M$. When $M$ is strictly pseudoconvex we denote by $\mathcal{P}_{+}$the set of positively oriented contact forms i.e. all $\theta \in \mathcal{P}$ such that $G_{\theta}$ is positive definite. If $M$ is nondegenerate then for each contact form $\theta \in \mathcal{P}$ there is a unique nowhere zero, globally defined, vector field $T \in \mathfrak{X}(M)$ (the Reeb vector field of $(M, \theta)$ ) such that $\theta(T)=1$ 
and $T\rfloor d \theta=0$. By taking into account the direct sum decomposition $T(M)=H(M) \oplus \mathbb{R} T$ one may extend the Levi form $G_{\theta}$ to a semi-Riemannian metric $g_{\theta}$ (the Webster metric of $(M, \theta)$ ) given by $g_{\theta}(X, Y)=G_{\theta}(X, Y)$, $g_{\theta}(X, T)=0$ and $g_{\theta}(T, T)=1$, for every $X, Y \in H(M)$. By a fundamental result of N. Tanaka, [37], and S. Webster, [38], for each contact form $\theta \in \mathcal{P}$ there is a unique linear connection $\nabla$ (the Tanaka-Webster connection of $(M, \theta)$ ) such that i) $H(M)$ is parallel with respect to $\nabla$, ii) $\nabla g_{\theta}=0$, $\nabla J=0$, and iii) the torsion tensor field $T_{\nabla}$ is pure i.e. $T_{\nabla}(Z, W)=0$, $T_{\nabla}(Z, \bar{W})=2 i G_{\theta}(Z, \bar{W}) T$ and $\tau \circ J+J \circ \tau=0$, for any $Z, W \in T_{1,0}(M)$. For all local calculations we consider a local frame $\left\{T_{\alpha}: 1 \leq \alpha \leq n\right\}$ of $T_{1,0}(M)$, defined on the open set $U$, and set

$$
\begin{gathered}
g_{\alpha \bar{\beta}}=G_{\theta}\left(T_{\alpha}, T_{\bar{\beta}}\right), \quad T_{\bar{\alpha}}=\bar{T}_{\alpha}, \quad \nabla T_{B}=\omega_{B}{ }^{A} T_{A}, \\
\omega_{B}{ }^{A}=\Gamma_{C B}^{A} \theta^{C}, \quad \tau\left(T_{\alpha}\right)=A_{\alpha}^{\bar{\beta}} T_{\bar{\beta}}, \quad A_{\alpha \beta}=g_{\alpha \bar{\gamma}} A_{\beta}^{\bar{\gamma}}, \\
\alpha, \beta, \gamma, \cdots \in\{1, \cdots, n\}, \quad A, B, C, \cdots \in\{0,1, \cdots, n, \overline{1}, \cdots, \bar{n}\} .
\end{gathered}
$$

Here $\left\{\theta^{\alpha}: 1 \leq \alpha \leq n\right\}$ is the adpated coframe determined by $\theta^{\alpha}\left(T_{\beta}\right)=\delta_{\beta}^{\alpha}$, $\theta^{\alpha}\left(T_{\bar{\beta}}\right)=0$ and $\theta^{\alpha}(T)=0$. Then (cf. e.g. (1.62) and (1.64) in [12], p. 39-40)

$$
d \theta=2 i g_{\alpha \bar{\beta}} \theta^{\alpha} \wedge \theta^{\bar{\beta}}, \quad d \theta^{\alpha}=\theta^{\beta} \wedge \omega_{\beta}{ }^{\alpha}+\theta \wedge \tau^{\alpha}, \quad A_{\alpha \beta}=A_{\beta \alpha},
$$

where $\tau^{\alpha} \equiv A_{\bar{\beta}}^{\alpha} \theta^{\bar{\beta}}$ and $A_{\bar{\beta}}^{\alpha}=\overline{A_{\beta}^{\bar{\alpha}}}$. Therefore, if we set $A(X, Y)=g_{\theta}(\tau X, Y)$ for any $X, Y \in \mathfrak{X}(M)$ then $A$ is symmetric. Let $R^{\nabla}$ be the curvature tensor field of the Tanaka-Webster connection $\nabla$. As to the local components of $R^{\nabla}$ we adopt the convention $R^{\nabla}\left(T_{B}, T_{C}\right) T_{A}=R_{A}{ }^{D}{ }_{B C} T_{D}$ (cf. [12], p. 50). The Ricci tensor of $\nabla$ is $\operatorname{Ric}_{\nabla}(Y, Z)=\operatorname{trace}\left\{X \in T(M) \longmapsto R^{\nabla}(X, Z) Y\right\}$ for any $Y, Z \in T(M)$. Locally we set $R_{A B}=\operatorname{Ric}_{\nabla}\left(T_{A}, T_{B}\right)$. The pseudohermitian Ricci tensor is then $R_{\lambda \bar{\mu}}$. By a result of S. Webster, [38] (to whom the notion is due) $R_{\lambda \bar{\mu}}=R_{\lambda}{ }^{\alpha} \alpha \bar{\mu}$. The pseudohermitian scalar curvature is $\rho=g^{\lambda \bar{\mu}} R_{\lambda \bar{\mu}}$ where $\left[g^{\alpha \bar{\beta}}\right]=\left[g_{\alpha \bar{\beta}}\right]^{-1}$. Let us set

$$
\Pi_{\alpha}{ }^{\beta}=d \omega_{\alpha}{ }^{\beta}-\omega_{\alpha}^{\gamma} \wedge \omega_{\gamma}{ }^{\beta}, \quad \Omega_{\alpha}{ }^{\beta}=\Pi_{\alpha}{ }^{\beta}-2 i \theta_{\alpha} \wedge \tau^{\beta}+2 i \tau_{\alpha} \wedge \theta^{\beta},
$$

where $\theta_{\alpha}=g_{\alpha \bar{\beta}} \theta^{\bar{\beta}}, \theta^{\bar{\alpha}}=\overline{\theta^{\alpha}}, \tau_{\alpha}=g_{\alpha \bar{\beta}} \tau^{\bar{\beta}}$ and $\tau^{\bar{\beta}}=A_{\alpha}^{\bar{\beta}} \theta^{\alpha}$. By a result of S.M. Webster, [38] (cf. also Theorem 1.7 in [12], p. 55)

$$
\Omega_{\alpha}{ }^{\beta}=R_{\alpha \lambda \bar{\mu}}{ }^{\beta} \theta^{\lambda} \wedge \theta^{\bar{\mu}}+W_{\alpha \lambda}^{\beta} \theta^{\lambda} \wedge \theta-W_{\alpha \bar{\lambda}}^{\beta} \theta^{\bar{\lambda}} \wedge \theta
$$

where $W_{\alpha \bar{\mu}}^{\beta}=g^{\beta \bar{\sigma}} \nabla_{\alpha} A_{\overline{\mu \sigma}}$ and $W_{\alpha \lambda}^{\beta}=g^{\beta \bar{\sigma}} \nabla_{\bar{\sigma}} A_{\alpha \lambda}$. Given $u \in C^{\infty}(M, \mathbb{R})$ the pseudohermitian Hessian is $\left(\nabla^{2} u\right)(X, Y)=\left(\nabla_{X} d u\right) Y$ for any $X, Y \in \mathfrak{X}(M)$. Locally we set $\nabla_{A} u_{B}=\left(\nabla^{2} u\right)\left(T_{A}, T_{B}\right)$. The pseudohermitian Hessian is not symmetric. Rather one has the commutation formulae

$$
\nabla_{\alpha} u_{\beta}=\nabla_{\beta} u_{\alpha}, \quad \nabla_{\alpha} u_{\bar{\beta}}=\nabla_{\bar{\beta}} u_{\alpha}-2 i g_{\alpha \bar{\beta}} u_{0}, \quad u_{0} \equiv T(u),
$$




$$
\nabla_{0} u_{\beta}=\nabla_{\beta} u_{0}-u_{\bar{\alpha}} A_{\beta}^{\bar{\alpha}} .
$$

The $3^{\text {rd }}$ order covariant derivative of $u$ is $\left(\nabla^{3} u\right)(X, Y, Z)=\left(\nabla_{X} H_{u}\right)(Y, Z)=$ $X\left(H_{u}(Y, Z)\right)-H_{u}\left(\nabla_{X} Y, Z\right)-H_{u}\left(Y, \nabla_{X} Z\right)$ for any $X, Y, Z \in \mathfrak{X}(M)$, where $H_{u} \equiv$ $\nabla^{2} u$. Locally we set $u_{A B C}=\left(\nabla^{3} u\right)\left(T_{A}, T_{B}, T_{C}\right)$. Commutation formulae for $u_{A B C}$ have been established by J.M. Lee, [30] (cf. also [12], p. 426) and are not needed through this paper. We shall use the divergence operator $\operatorname{div}: \mathfrak{X}(M) \rightarrow C^{\infty}(M, \mathbb{R})$ determined by $\mathcal{L}_{X} \Psi_{\theta}=\operatorname{div}(X) \Psi_{\theta}$ for every $X \in \mathfrak{X}(M)$, where $\mathcal{L}_{X}$ is the Lie derivative. The horizontal gradient of $u \in C^{1}(M, \mathbb{R})$ is $\nabla^{H} u=\Pi_{H} \nabla u$ where $\Pi_{H}: T(M) \rightarrow H(M)$ is the projection associated to the direct sum decomposition $T(M)=H(M) \oplus \mathbb{R} T$ and $\nabla u$ is the ordinary semi-Riemannian gradient of $u$ with respect to $g_{\theta}$ i.e. $g_{\theta}(\nabla u, X)=X(u)$ for any $X \in \mathfrak{X}(M)$. The sublaplacian of $(M, \theta)$ is the second order differential operator $\Delta_{b} u=-\operatorname{div}\left(\nabla^{H} u\right), u \in C^{2}(M, \mathbb{R})$. Another useful expression of the sublaplacian is $\Delta_{b} u=-\operatorname{trace}_{G_{\theta}} \Pi_{H} \nabla^{2} u$ or $\Delta_{b} u=-\sum_{a=1}^{2 n}\left\{E_{a}\left(E_{a}(u)\right)-\left(\nabla_{E_{a}} E_{a}\right)(u)\right\}$ for any local $G_{\theta}$-orthonormal frame $\left\{E_{a}: 1 \leq a \leq 2 n\right\}$ of $H(M)$ on $U \subset M$. If $\left\{T_{\alpha}: 1 \leq \alpha \leq n\right\}$ is a local frame of $T_{1,0}(M)$ on $U \subset M$ then

$$
\Delta_{b} u=-\nabla_{\alpha} u^{\alpha}-\nabla_{\bar{\alpha}} u^{\bar{\alpha}} .
$$

A complex valued differential $p$-form $\omega \in \Omega^{p}(M) \otimes \mathbb{C}$ is a $(p, 0)$-form (respectively a $(0, p)$-form) if $\left.T_{0,1}(M)\right\rfloor \omega=0$ (respectively $\left.T_{0,1}(M)\right\rfloor \omega=0$ and $T\rfloor \omega=0)$. Let $\Lambda^{p, 0}(M) \rightarrow M$ and $\Lambda^{0, p}(M) \rightarrow M$ be the relevant bundles and $\Omega^{p, 0}(M)$ and $\Omega^{0, p}(M)$ the corresponding spaces of sections. Let $\mathcal{F}$ be the flow on $M$ tangent to the Reeb vector $T$ (i.e. $T(\mathcal{F})=\mathbb{R} T$ ). Let $\left.\Omega_{B}^{1,0}(\mathcal{F})=\left\{\omega \in \Omega^{1,0}(M): T\right\rfloor \omega=0\right\}$ be the space of all basic $(1,0)$ forms (on the foliated manifold $(M, \mathcal{F})$, cf. also [5]). If $\omega \in \Omega_{B}^{1,0}(\mathcal{F})$ one may use the Levi form to define a unique complex vector field $\omega^{\sharp} \in$ $C^{\infty}\left(T_{0,1}(M)\right)$. Here $\omega^{\sharp}$ is determined by $\omega(Z)=G_{\theta}\left(Z, \omega^{\sharp}\right)$ for any $Z \in$ $T_{1,0}(M)$ hence locally $\omega^{\sharp}=\omega^{\bar{\beta}} T_{\bar{\beta}}$ where $\omega^{\bar{\beta}}=g^{\alpha \bar{\beta}} \omega_{\alpha}$ and $\omega=\omega_{\alpha} \theta^{\alpha}$. Let $\delta_{b}: \Omega_{B}^{1,0}(\mathcal{F}) \rightarrow C^{\infty}(M, \mathbb{C}$ ) be the differential operator (due to [30]) defined by $\delta_{b} \omega=\operatorname{div}\left(\omega^{\sharp}\right)$ and $\delta_{b} \theta=0$ for any $\omega \in \Omega_{B}^{0,1}(\mathcal{F})$. Similarly if $\eta \in \Omega^{0,1}(M)$ then let $\eta^{\sharp} \in C^{\infty}\left(T_{1,0}(M)\right)$ be determined by $\eta(\bar{Z})=G_{\theta}\left(\eta^{\sharp}, \bar{Z}\right), Z \in T_{1,0}(M)$, and let us consider

$$
\bar{\delta}_{b}: \Omega^{0,1}(M) \rightarrow C^{\infty}(M, \mathbb{C}), \quad \bar{\delta}_{b} \eta=\operatorname{div}\left(\eta^{\sharp}\right), \quad \eta \in \Omega^{0,1}(M),
$$

so that (locally) $\eta^{\sharp}=\eta^{\alpha} T_{\alpha}$ where $\eta=\eta_{\bar{\beta}} \theta^{\bar{\beta}}$ and $\eta^{\alpha}=g^{\alpha \bar{\beta}} \eta_{\bar{\beta}}$. Also $\delta_{b} \omega=$ $\nabla_{\bar{\beta}} \omega^{\bar{\beta}}$ and $\bar{\delta}_{b} \eta=\nabla_{\alpha} \eta^{\alpha}$. For each $f \in C^{\infty}(M, \mathbb{C})$ we set

$$
(P f) Z=g^{\alpha \bar{\beta}}\left(\nabla^{3} f\right)\left(Z, T_{\alpha}, T_{\bar{\beta}}\right)+2 n i A\left(Z,\left(\nabla^{H} f\right)^{1,0}\right),
$$




$$
(P f) \bar{Z}=0, \quad(P f) T=0, \quad Z \in T_{1,0}(M) .
$$

Here $X^{1,0}=\Pi_{1,0} X$ for any $X \in H(M)$ and $\Pi_{1,0}: H(M) \otimes \mathbb{C} \rightarrow T_{1,0}(M)$ is the natural projection associated to $H(M) \otimes \mathbb{C}=T_{1,0}(M) \oplus T_{0,1}(M)$. Note that $g^{\alpha \bar{\beta}}\left(\nabla_{T_{\bar{\beta}}}\left(\nabla^{2} f\right)\right)\left(T_{\alpha}, Z\right)$ is invariant under a transformation $T_{\alpha}^{\prime}=U_{\alpha}^{\beta} T_{\beta}$ with $\operatorname{det}\left[U_{\alpha}^{\beta}\right] \neq 0$ on $U \cap U^{\prime}$, hence $(P f) Z$ is globally defined. Locally one has

$$
P f=\left(P_{\beta} f\right) \theta^{\beta}, \quad P_{\beta} f=f_{\beta}^{\bar{\alpha}}{ }_{\bar{\alpha}}+2 n i A_{\beta \gamma} f^{\gamma},
$$

(compare to Definition 1.1 and (1.2) in [10], p. 263). Similar to $P$ : $C^{\infty}(M, \mathbb{C}) \rightarrow \Omega_{B}^{1,0}(\mathcal{F})$ we build $\bar{P}: C^{\infty}(M, \mathbb{C}) \rightarrow \Omega^{0,1}(M)$ given by

$$
\begin{gathered}
(\bar{P} f) \bar{Z}=g^{\alpha \bar{\beta}}\left(\nabla^{3} f\right)\left(Z, T_{\bar{\beta}}, T_{\alpha}\right)-2 n i A\left(\bar{Z},\left(\nabla^{H} f\right)^{0,1}\right), \\
(\bar{P} f) Z=0, \quad(\bar{P} f) T=0, \quad Z \in T_{1,0}(M),
\end{gathered}
$$

where $X^{0,1}=\overline{X^{1,0}}$ for any $X \in H(M)$. Also let ${ }^{1}$

$$
P_{0} f=\delta_{b}(P f)+\bar{\delta}_{b}(\bar{P} f), \quad f \in C^{\infty}(M, \mathbb{C}) .
$$

From now on we assume that $M$ is a compact strictly pseudoconvex CR manifold and $\theta \in \mathcal{P}_{+}$. Then $g_{\theta}$ is a Riemannian metric on $M$. It should be observed that the operators above are complexifications of real operators familiar in Riemannian geometry, as follows. For instance let $\sharp$ be "raising of indices" with respect to $g_{\theta}$ i.e. $g_{\theta}\left(\alpha^{\sharp}, X\right)=\alpha(X)$ for any (real) 1 -form $\eta \in \Omega^{1}(M)$ and any (real) vector field $X \in \mathfrak{X}(M)$. Then the musical isomorphisms $\sharp: \Omega_{B}^{1,0}(\mathcal{F}) \rightarrow C^{\infty}\left(T_{0,1}(M)\right)$ and $\sharp: \Omega^{0,1}(M) \rightarrow C^{\infty}\left(T_{1,0}(M)\right)$ (as built above) are restrictions of the $\mathbb{C}$-linear extension (to $\Omega^{1}(M) \otimes \mathbb{C}=$ $\left.C^{\infty}\left(T^{*}(M) \otimes \mathbb{C}\right)\right)$ of $\sharp: \Omega^{1}(M) \rightarrow \mathfrak{X}(M)$ to $\Omega_{B}^{1,0}(\mathcal{F})$ and $\Omega^{0,1}(M)$ respectively. Also let $\Omega_{B}^{1}(\mathcal{F})$ be the space of all basic 1 -forms on $(M, \mathcal{F})$ and $d_{b}: C^{\infty}(M) \rightarrow \Omega_{B}^{1}(\mathcal{F})$ the first order differential operator given by $d_{b} u=$ $d u-u_{0} \theta$ for every $u \in C^{\infty}(M, \mathbb{R})$ where $u_{0} \equiv T(u)$. Let $d_{b}^{*}$ be the formal adjoint of $d_{b}$ i.e. $\left(d_{b}^{*} \omega, u\right)_{L^{2}}=\left(\omega, d_{b} u\right)_{L^{2}}, \omega \in \Omega_{B}^{1}(\mathcal{F}), u \in C^{\infty}(M)$, with respect to the $L^{2}$ inner products

$$
(u, v)_{L^{2}}=\int_{M} u v \Psi_{\theta}, \quad(\alpha, \beta)_{L^{2}}=\int_{M} g_{\theta}^{*}(\alpha, \beta) \Psi_{\theta},
$$

for any $u, v \in C^{\infty}(M, \mathbb{R})$ and $\alpha, \beta \in \Omega^{1}(M)$. Let $d_{b}: C^{\infty}(M, \mathbb{C}) \rightarrow \Omega_{B}^{1}(\mathcal{F}) \otimes \mathbb{C}$ and $d_{b}^{*}: \Omega_{B}^{1}(\mathcal{F}) \otimes \mathbb{C} \rightarrow C^{\infty}(M, \mathbb{C})$ be the $\mathbb{C}$-linear extensions of $d_{b}$ and $d_{b}^{*}$. Then

Lemma 1. i) $\Omega_{B}^{1}(\mathcal{F}) \otimes \mathbb{C}=\Omega_{B}^{1,0}(\mathcal{F}) \oplus \Omega^{0,1}(M)$, ii) $d_{b} f=\partial_{b} f+\bar{\partial}_{b} f$ for any $f \in C^{\infty}(M, \mathbb{C})$, iii) $\left.d_{b}^{*}\right|_{\Omega_{B}^{1,0}(\mathcal{F})}=\partial_{b}^{*}=-\delta_{b}$, iv) $\left.d_{b}^{*}\right|_{\Omega^{0,1}(M)}=\bar{\partial}_{b}^{*}=-\bar{\delta}_{b}$.

\footnotetext{
${ }^{1}$ The operator $P_{0}$ in this paper and [10] differ by a multiplicative factor $\frac{1}{4}$.
} 
Here the tangential C-R operator $\bar{\partial}_{b}$ is thought of as $\Omega^{0,1}(M)$-valued (i.e. one requests that $Z\rfloor \bar{\partial}_{b} f=$ and $\left.T\right\rfloor \bar{\partial}_{b} f=0$ to start with). Also $\partial_{b} f$ is the unique element of $\Omega_{B}^{1,0}(\mathcal{F})$ coinciding with $d f$ on $T_{1,0}(M)$. Locally $\partial_{b} f=$ $f_{\alpha} \theta^{\alpha}$ and $\bar{\partial}_{b} f=f_{\bar{\alpha}} \theta^{\bar{\alpha}}$ where $f_{\alpha} \equiv T_{\alpha}(f)$ and $f_{\bar{\alpha}} \equiv T_{\bar{\alpha}}(f)$. Also $\partial_{b}^{*}$ and $\bar{\partial}_{b}^{*}$ are the formal adjoints of $\partial_{b}: C^{\infty}(M, \mathbb{C}) \rightarrow \Omega_{B}^{1,0}(\mathcal{F})$ and $\bar{\partial}_{b}: C^{\infty}(M, \mathbb{C}) \rightarrow$ $\Omega^{0,1}(M)$ with respect to the $L^{2}$ inner products

$$
(f, g)_{L^{2}}=\int_{M} f \bar{g} \Psi_{\theta}, \quad\left(\omega_{1}, \omega_{2}\right)_{L^{2}}=\int_{M} G_{\theta}^{*}\left(\omega_{1}, \bar{\omega}_{2}\right) \Psi_{\theta},
$$

for any $f, g \in C^{\infty}(M, \mathbb{C})$ and any complex 1-forms $\omega_{1}, \omega_{2}$ either in $\Omega_{B}^{1,0}(\mathcal{F})$ or in $\Omega^{0,1}(M)$. Statements (i)-(ii) in Lemma 1 are immediate. The last equality in (iii) (respectively in (iv)) is due to [30] (cf. also [12], p. 280). To prove (iii) one integrates by parts in $\left(d_{b}^{*} \omega, f\right)_{L^{2}}$. For every $f \in C^{\infty}(M, \mathbb{R})$

$$
\int_{M} g_{\theta}^{*}\left((P+\bar{P}) f, \overline{d_{b} f}\right) \Psi_{\theta}=\left(P f+\bar{P} f, d_{b} f\right)_{L^{2}}=-\left(P_{0} f, f\right)_{L^{2}}
$$

(compare to (1.3) in [10], p. 263). By a result of S-C. Chang \& H-L. Chiu, [10], the operator $P_{0}$ is nonnegative i.e. $\int_{M}\left(P_{0} u\right) u \Psi_{\theta} \geq 0$ for any $u \in$ $C^{\infty}(M, \mathbb{R})$. We end the preparation of $\mathrm{CR}$ and pseudohermitian geometry by stating the identity (a straightforward consequence of (14))

$$
\begin{aligned}
& u^{\alpha} u_{\alpha}{ }_{\beta}{ }+u^{\bar{\alpha}} u_{\bar{\alpha}}^{\bar{\beta}} \bar{\beta}=-u^{\alpha} P_{\alpha} u-u^{\bar{\alpha}} P_{\bar{\alpha}} u+ \\
+ & 2 n i\left(A_{\alpha \beta} u^{\alpha} u^{\beta}-A_{\bar{\alpha} \bar{\beta}} u^{\bar{\alpha}} u^{\bar{\beta}}\right)-\left(\nabla^{H} u\right)\left(\Delta_{b} u\right) .
\end{aligned}
$$

Compare to (2.3) in [10], p. 267.

\section{BochNer-Lichnerowicz FORMULAE ON FefFERMAN SPACES}

Let $S^{1} \rightarrow C(M) \stackrel{\pi}{\rightarrow} M$ be the canonical circle bundle over a strictly pseudoconvex CR manifold $M$, of CR dimension $n$ (cf. e.g. Definition 2.9 in [12], p. 119). We set $\mathfrak{M}=C(M)$ for simplicity. Let $\theta \in \mathcal{P}_{+}$be a positively oriented contact form on $M$ and let $F_{\theta}$ be the corresponding Fefferman metric on $\mathfrak{M}$ i.e.

$$
\begin{gathered}
F_{\theta}=\pi^{*} \tilde{G}_{\theta}+2\left(\pi^{*} \theta\right) \odot \sigma \\
\sigma=\frac{1}{n+2}\left\{d \gamma+\pi^{*}\left(i \omega_{\alpha}{ }^{\alpha}-\frac{i}{2} g^{\mu \bar{\nu}} d g_{\mu \bar{\nu}}-\frac{\rho}{4(n+1)} \theta\right)\right\} .
\end{gathered}
$$

Cf. Definition 2.15 and Theorem 2.4 in [12], p. 128-129. As to the notations in (19)-(20) we set $\tilde{G}_{\theta}=G_{\theta}$ on $H(M) \otimes H(M)$ and $\tilde{G}_{\theta}(T, W)=0$ for every $W \in \mathfrak{X}(M)$. Moreover $\gamma$ is a local fibre coordinate on $\mathfrak{M}$. We recall that $F_{\theta} \in \operatorname{Lor}(\mathfrak{M})$ i.e. $F_{\theta}$ is a Lorentzian metric on $\mathfrak{M}$ (a semi-Riemannian metric of signature $(-+\cdots+))$. 
Let $D$ be the Levi-Civita connection of $\left(\mathfrak{M}, F_{\theta}\right)$. Given a point $z_{0} \in \mathfrak{M}$ let $\left\{E_{p}: 1 \leq p \leq 2 n+2\right\}$ be a local orthonormal (i.e. $F_{\theta}\left(E_{p}, E_{q}\right)=$ $\epsilon_{p} \delta_{p q}$ with $\left.\epsilon_{p} \in\{ \pm 1\}\right)$ frame of $T(\mathfrak{M})$, defined on an open neighborhood $\pi^{-1}(U) \subset \mathfrak{M}$ of $z_{0}$, such that $\left(D_{E_{p}} E_{q}\right)\left(z_{0}\right)=0$ for any $1 \leq p, q \leq 2 n+2$. Such a local frame may always be built by parallel translating a given orthonormal basis $\left\{e_{p}: 1 \leq p \leq 2 n+2\right\} \subset T_{z_{0}}(\mathfrak{M})$ along the geodesics of $\left(\mathfrak{M}, F_{\theta}\right)$ issuing at $z_{0}$. Let $\square$ be the wave operator (the Laplace-Beltrami operator of $\left.\left(\mathfrak{M}, F_{\theta}\right)\right)$. If $f \in C^{\infty}(\mathfrak{M}, \mathbb{R})$ and $g=F_{\theta}(D f, D f)$ then $\square g=$ $-\sum_{p=1}^{2 n+2} \epsilon_{p}\left\{E_{p}\left(E_{p}(g)\right)-\left(D_{E_{p}} E_{p}\right)(g)\right\}$. A calculation of $(\square g)\left(z_{0}\right)$, merely adapting the proof of (G.IV.5) in [8], p. 131, to Lorentzian signature, leads to

(21) $-\frac{1}{2} \square\left(F_{\theta}(D f, D f)\right)=F_{\theta}^{*}\left(D^{2} f, D^{2} f\right)-(D f)(\square f)+\operatorname{Ric}_{D}(D f, D f)$.

Here $\operatorname{Ric}_{D}(X, Y)=\operatorname{trace}\left\{Z \in T(\mathfrak{M}) \mapsto R^{D}(Z, Y) X\right\}$ and $R^{D}$ is the curvature tensor field of $D$. Let us assume that $M$ is a closed manifold (i.e. $M$ is compact and $\partial M=\emptyset$ ). Then $\mathfrak{M}$ is a closed manifold, as well (as the total space of a locally trivial bundle over a compact manifold, with compact fibres). Integration of (21) over $\mathfrak{M}$ leads (by Green's lemma) to the (Lorentzian analog to the) $L^{2}$ Bochner-Lichnerowicz formula

$$
\int_{\mathfrak{M}}\left\{F_{\theta}^{*}\left(D^{2} f, D^{2} f\right)+\operatorname{Ric}_{D}(D f, D f)-(D f)(\square f)\right\} d \operatorname{vol}\left(F_{\theta}\right)=0 .
$$

\section{Curvature theory}

By a result in [21] the 1-form $\sigma \in \Omega^{1}(M)$ is a connection form in the canonical circle bundle $S^{1} \rightarrow \mathfrak{M} \rightarrow M$. Let $X^{\uparrow} \in \mathfrak{X}(\mathfrak{M})$ denote the horizontal lift of $X \in \mathfrak{X}(M)$ i.e. $X_{z}^{\uparrow} \in \operatorname{Ker}\left(d_{z} \pi\right)$ and $\left(d_{z} \pi\right) X_{z}^{\uparrow}=X_{\pi(z)}$ for any $z \in \mathfrak{M}$. Let $S \in \mathfrak{X}(\mathfrak{M})$ be the tangent to the $S^{1}$-action i.e. locally $S=[(n+2) / 2] \partial / \partial \gamma$. The Levi-Civita connection $D$ of $\left(\mathfrak{M}, F_{\theta}\right)$ is given by (cf. Lemma 2 in [6], p. 03504-26)

$$
\begin{gathered}
D_{X^{\uparrow}} Y^{\uparrow}=\left(\nabla_{X} Y\right)^{\uparrow}+\{\Omega(X, Y) \circ \pi\} T^{\uparrow}+\left\{\sigma\left(\left[X^{\uparrow}, Y^{\uparrow}\right]\right)-2 A(X, Y) \circ \pi\right\} S, \\
D_{X^{\uparrow}} T^{\uparrow}=\{\tau(X)+\phi(X)\}^{\uparrow}, \\
D_{T^{\uparrow}} X^{\uparrow}=\left(\nabla_{T} X+\phi X\right)^{\uparrow}+4(d \sigma)\left(X^{\uparrow}, T^{\uparrow}\right) S, \\
D_{X^{\uparrow}} S=D_{S} X^{\uparrow}=\frac{1}{2}(J X)^{\uparrow}, \\
D_{T^{\uparrow}} T^{\uparrow}=2 V^{\uparrow}, \quad D_{S} S=D_{S} T^{\uparrow}=D_{T^{\uparrow}} S=0,
\end{gathered}
$$


where $\Omega=-d \theta$ while $\phi: H(M) \rightarrow H(M)$ and $V \in H(M)$ are the bundle endomorphism and vector field determined by

$$
G_{\theta}(\phi X, Y) \circ \pi=(d \sigma)\left(X^{\uparrow}, Y^{\uparrow}\right), \quad G_{\theta}(V, X)=(d \sigma)\left(T^{\uparrow}, X^{\uparrow}\right),
$$

for any $X, Y \in H(M)$. Locally $\phi$ and $V$ are given by

$$
\begin{aligned}
\phi_{\alpha}{ }^{\beta} & =\frac{i}{2(n+2)}\left\{R_{\alpha}{ }^{\beta}-\frac{\rho}{2(n+1)} \delta_{\alpha}^{\beta}\right\}, \quad \phi_{\alpha}{ }^{\bar{\beta}}=0, \quad \phi_{\alpha}{ }^{0}=0, \\
V^{\alpha} & =g^{\alpha \bar{\beta}} V_{\bar{\beta}}, \quad V_{\bar{\beta}}=\frac{1}{2(n+2)}\left\{\frac{1}{4(n+1)} \rho_{\bar{\beta}}+i W_{\alpha \bar{\beta}}^{\alpha}\right\} .
\end{aligned}
$$

In particular $[J, \phi]=0$ (as a consequence of (29)). We recall (cf. (1.100) in [12], p. 58)

$$
\begin{gathered}
\operatorname{Ric}_{g_{\theta}}\left(T_{\mu}, T_{\bar{v}}\right)=-\frac{1}{2} R_{\mu \bar{\nu}}+g_{\mu \bar{\nu}}, \\
R_{\mu \nu}=i(n-1) A_{\mu \nu}, \quad R_{0 v}=S_{\bar{\mu} \nu}^{\bar{\mu}}, \quad R_{\mu 0}=0, \quad R_{00}=0 .
\end{gathered}
$$

Here $\operatorname{Ric}_{g_{\theta}}$ is the Ricci curvature of $\left(M, g_{\theta}\right)$. Also $S(X, Y)=\left(\nabla_{X} \tau\right) Y-$ $\left(\nabla_{Y} \tau\right) X$ for any $X, Y \in \mathfrak{X}(M)$, so that $S_{\bar{\mu} \nu}^{\bar{\mu}}$ are among $S_{k \ell}^{j} T_{j}=S\left(T_{k}, T_{\ell}\right)$. As a consequence of (31) one has $R_{\mu \bar{\nu}}=R_{\bar{v} \mu}$. Take the derivative of (20)

$$
(n+2) d \sigma=\pi^{*}\left\{i d \omega_{\alpha}{ }^{\alpha}-\frac{i}{2} d g^{\mu \bar{\nu}} \wedge d g_{\mu \bar{\nu}}-\frac{1}{4(n+1)} d(\rho \theta)\right\}
$$

and observe that $d g^{\mu \bar{v}} \wedge d g_{\mu \bar{v}}=0$. Also (by Theorem 1.7 in [12], p. 55)

$$
d \omega_{\alpha}^{\alpha}=R_{\mu \bar{v}} \theta^{\mu} \wedge \theta^{\bar{v}}+\left(W_{\alpha \lambda}^{\alpha} \theta^{\lambda}-W_{\alpha \bar{\mu}}^{\alpha} \theta^{\bar{\mu}}\right) \wedge \theta .
$$

By (31)-(32)

$$
\operatorname{Ric}_{\nabla}(X, J Y)=-2 i\left(R_{\mu \bar{v}} \theta^{\mu} \wedge \theta^{\bar{v}}\right)(X, Y)-(n-1) A(X, Y)
$$

for any $X, Y \in H(M)$. Also $d(\rho \theta)=-\rho \Omega$ on $H(M) \otimes H(M)$. Consequently

$$
\begin{gathered}
2(d \sigma)\left(X^{\uparrow}, Y^{\uparrow}\right)=\frac{1}{n+2}\left\{\frac{\rho}{2(n+1)} \Omega(X, Y)-\right. \\
\left.-(n-1) A(X, Y)-\operatorname{Ric}_{\nabla}(X, J Y)\right\} .
\end{gathered}
$$

By a result in [28], Vol. I, p. 65, $[X, Y]^{\uparrow}$ is the horizontal component of $\left[X^{\uparrow}, Y^{\uparrow}\right]$ for any $X, Y \in \mathfrak{X}(M)$. When $X, Y \in H(M)$ the vertical component may be easily derived from (34). One obtains the decomposition

$$
\begin{aligned}
& {\left[X^{\uparrow}, Y^{\uparrow}\right]=[X, Y]^{\uparrow}+\frac{2}{n+2}\left\{\operatorname{Ric}_{\nabla}(X, J Y)+\right.} \\
& \left.+(n-1) A(X, Y)-\frac{\rho}{2(n+1)} \Omega(X, Y)\right\} S .
\end{aligned}
$$


Similarly let us compute $f \in C^{\infty}(M)$ in $\left[X^{\uparrow}, T^{\uparrow}\right]=[X, T]^{\uparrow}+f S$. If $\varphi=$ $i\left(W_{\alpha \lambda}^{\alpha} \theta^{\lambda}-W_{\alpha \bar{\mu}}^{\alpha} \theta^{\bar{\mu}}\right)$ then

or

$$
\begin{gathered}
i\left(d \omega_{\alpha}{ }^{\alpha}\right)(X, T)=(\varphi \wedge \theta)(X, T)=\frac{1}{2} \varphi(X), \\
2(n+2)(d \sigma)\left(X^{\uparrow}, T^{\uparrow}\right)=\varphi(X)-\frac{1}{2(n+1)} d(\rho \theta)(X, T)
\end{gathered}
$$

$$
2(d \sigma)\left(X^{\uparrow}, T^{\uparrow}\right)=\frac{1}{n+2}\left\{\varphi(X)-\frac{1}{4(n+1)} X(\rho)\right\}
$$

as $T\rfloor d \theta=0$. We conclude (as $\sigma(S)=\frac{1}{2}$ )

$$
\left[X^{\uparrow}, T^{\uparrow}\right]=[X, T]^{\uparrow}+\frac{2}{n+2}\left\{\frac{1}{4(n+1)} X(\rho)-\varphi(X)\right\} S .
$$

Lemma 2. Let $M$ be a strictly pseudoconvex $C R$ manifold, of CR dimension $n$, and $\theta \in \mathcal{P}_{+}$a positively oriented contact form. The curvature $R^{D}$ of the Lorentzian manifold $\left(\mathfrak{M}, F_{\theta}\right)$ is given by

$$
\begin{gathered}
R^{D}\left(X^{\uparrow}, Y^{\uparrow}\right) Z^{\uparrow}=\left(R^{\nabla}(X, Y) Z\right)^{\uparrow}- \\
-\frac{1}{2(n+1)(n+2)}\{X(\rho) \Omega(Y, Z)-Y(\rho) \Omega(X, Z)\} S- \\
-\frac{n+5}{n+2}\left\{\left(\nabla_{X} A\right)(Y, Z)-\left(\nabla_{Y} A\right)(X, Z)\right\} S+ \\
+\frac{1}{n+2}\left\{\left(\nabla_{X} \operatorname{Ric}_{\nabla}\right)(Y, J Z)-\left(\nabla_{Y} \operatorname{Ric}_{\nabla}\right)(Y, J Z)\right\} S+ \\
+\Omega(Y, Z)\left\{(\tau X)^{\uparrow}+(\phi X)^{\uparrow}-\frac{\rho}{4(n+1)(n+2)}(J X)^{\uparrow}\right\}- \\
-\Omega(X, Z)\left\{(\tau Y)^{\uparrow}+(\phi Y)^{\uparrow}-\frac{\rho}{4(n+1)(n+2)}(J Y)^{\uparrow}\right\}+ \\
+\frac{1}{2(n+2)}\left\{\operatorname{Ric}_{\nabla}(Y, J Z)-(n+5) A(Y, Z)\right\}(J X)^{\uparrow}- \\
-\frac{1}{2(n+2)}\left\{\operatorname{Ric}_{\nabla}(X, J Z)-(n+5) A(X, Z)\right\}(J Y)^{\uparrow}- \\
-\frac{1}{n+2}\left\{\operatorname{Ric}_{\nabla}(X, J Y)(J Z)^{\uparrow}-2 \Omega(X, Y) \operatorname{Ric}(T, J Z) S\right\}- \\
-\frac{1}{n+2}\left\{(n-1) A(X, Y)-\frac{\rho}{2(n+1)} \Omega(X, Y)\right\}(J Z)^{\uparrow}- \\
-2 \Omega(X, Y)\left\{(\phi Z)^{\uparrow}+\frac{2}{n+2}\left[\varphi(Z)-\frac{1}{4(n+1)} Z(\rho)\right] S\right\} .
\end{gathered}
$$




$$
\begin{gathered}
R^{D}\left(X^{\uparrow}, T^{\uparrow}\right) Z^{\uparrow}=\left(R^{\nabla}(X, T) Z\right)^{\uparrow}+\left(\left(\nabla_{X} \phi\right) Z\right)^{\uparrow}+ \\
+\frac{1}{n+2}\left\{\varphi(Z)(J X)^{\uparrow}+\varphi(X)(J Z)^{\uparrow}-\left[\operatorname{Ric}_{\nabla}(X, J \phi Z)+\operatorname{Ric}_{\nabla}(\tau X, J Z)\right] S\right\}- \\
-\frac{1}{4(n+1)(n+2)}\left\{Z(\rho)(J X)^{\uparrow}+X(\rho)(J Z)^{\uparrow}\right\}+ \\
+\frac{2}{n+2}\left\{\left(\nabla_{X} \varphi\right) Z-\frac{1}{4(n+1)}\left(\nabla_{X} d \rho\right) Z\right\} S- \\
-\frac{1}{n+2}\left\{\left(\nabla_{T} \operatorname{Ric}_{\nabla}\right)(X, J Z)-(n+5)\left(\nabla_{T} A\right)(X, Z)\right\} S+ \\
+\{\Omega(X, \phi Z)-\Omega(\tau X, Z)\}\left\{T^{\uparrow}-\frac{\rho}{2(n+1)(n+2)} S\right\}- \\
-2 \Omega(X, Z)\left\{V^{\uparrow}-\frac{T(\rho)}{4(n+1)(n+2)} S\right\}-\frac{3(n+3)}{n+2}\{A(X, \phi Z)-A(\tau X, Z)\} S,
\end{gathered}
$$

$$
\begin{gathered}
R^{D}\left(X^{\uparrow}, S\right) Z^{\uparrow}=-\frac{1}{2(n+2)}\left\{\operatorname{Ric}_{\nabla}(X, Z)+(n+5) A(X, J Z)\right\} S- \\
-\frac{1}{2} G_{\theta}(X, Z)\left\{T^{\uparrow}-\frac{\rho}{2(n+1)(n+2)} S\right\},
\end{gathered}
$$

$-\frac{1}{n+2}\left\{\operatorname{Ric}_{\nabla}(J \tau X, Y)-\operatorname{Ric}_{\nabla}(X, J \tau Y)+\operatorname{Ric}_{\nabla}(J \phi X, Y)-\operatorname{Ric}_{\nabla}(X, J \phi Y)\right\} S-$

$$
-\frac{n+5}{2(n+2)^{2}}\left\{\operatorname{Ric}_{\nabla}(\tau X, J Y)-\operatorname{Ric}_{\nabla}(J X, \tau Y)+2(n-1) \Omega(\tau X, \tau Y)\right\},
$$

$$
\begin{aligned}
& R^{D}\left(X^{\uparrow}, Y^{\uparrow}\right) S=0, \quad R^{D}\left(T^{\uparrow}, S\right) T^{\uparrow}=0, \quad R^{D}\left(T^{\uparrow}, S\right) S=0, \\
& R^{D}\left(T^{\uparrow}, S\right) Z^{\uparrow}= \\
= & \frac{1}{n+2}\left\{\varphi(J Z)-2 \varphi(Z)-\frac{1}{4(n+1)}[(J Z)(\rho)-2 Z(\rho)]\right\} S,
\end{aligned}
$$

for any $X, Y, Z \in H(M)$.

Proof. As $H(M)$ is parallel with respect to $\nabla$ one has $\nabla_{Y} Z \in H(M)$. Then (by (23) and (34))

$$
\begin{gathered}
D_{X^{\uparrow}}\left(\nabla_{Y} Z\right)^{\uparrow}=\left(\nabla_{X} \nabla_{Y} Z\right)^{\uparrow}+\Omega\left(X, \nabla_{Y} Z\right)\left\{T^{\uparrow}-\frac{\rho}{2(n+1)(n+2)} S\right\}+ \\
+\frac{1}{n+2}\left\{\operatorname{Ric}_{\nabla}\left(X, J \nabla_{Y} Z\right)-(n+5) A\left(X, \nabla_{Y} Z\right)\right\} S .
\end{gathered}
$$

Next (by (23)-(24), (26), (34) and (44))

$$
D_{X^{\uparrow}} D_{Y^{\uparrow}} Z^{\uparrow}=\left(\nabla_{X} \nabla_{Y} Z\right)^{\uparrow}+
$$




$$
\begin{gathered}
+\left\{X(\Omega(Y, Z))+\Omega\left(X, \nabla_{Y} Z\right)\right\}\left\{T^{\uparrow}-\frac{\rho}{2(n+1)(n+2)} S\right\}+ \\
-\frac{X(\rho)}{2(n+1)(n+2)} \Omega(Y, Z) S-\frac{n+5}{n+2}\left\{X(A(Y, Z))+A\left(X, \nabla_{Y} Z\right)\right\} S+ \\
+\frac{1}{n+2}\left\{X\left(\operatorname{Ric}_{\nabla}(Y, J Z)\right)+\operatorname{Ric}_{\nabla}\left(X, J \nabla_{Y} Z\right)\right\} S+ \\
+\Omega(Y, Z)\left\{(\tau X)^{\uparrow}+(\phi X)^{\uparrow}-\frac{\rho}{4(n+1)(n+2)}(J X)^{\uparrow}\right\}+ \\
+\frac{1}{2(n+2)}\left\{\operatorname{Ric}_{\nabla}(Y, J Z)-(n+5) A(Y, Z)\right\}(J X)^{\uparrow} .
\end{gathered}
$$

The calculation of $D_{\left[X^{\uparrow}, Y^{\uparrow}\right]} Z^{\uparrow}$ is a bit trickier as $[X, Y] \notin H(M)$ in general. To start with one uses the decomposition (35) followed by $[X, Y]=\Pi_{H}[X, Y]+$ $\theta([X, Y]) T$. This yields (by (26))

$$
\begin{gathered}
D_{\left[X^{\uparrow}, Y^{\uparrow}\right]} Z^{\uparrow}=D_{[X, Y]^{\uparrow}} Z^{\uparrow}+\frac{2}{n+2} B(X, Y) D_{S} Z^{\uparrow}= \\
=D_{\left(\Pi_{H}[X, Y]\right)^{\uparrow}} Z^{\uparrow}+\theta([X, Y]) D_{T^{\uparrow}} Z^{\uparrow}+\frac{1}{n+2} B(X, Y)(J Z)^{\uparrow}
\end{gathered}
$$

where we have set

$$
B(X, Y)=\operatorname{Ric}_{\nabla}(X, J Y)+(n-1) A(X, Y)-\frac{\rho}{2(n+1)} \Omega(X, Y)
$$

for simplicity. At this point we may use (23) (as $\Pi_{H}[X, Y] \in H(M)$ ) and (25) so that

$$
\begin{gathered}
D_{\left[X^{\uparrow}, Y^{\uparrow}\right]} Z^{\uparrow}=\left(\nabla_{\Pi_{H}[X, Y]} Z\right)^{\uparrow}+\Omega\left(\Pi_{H}[X, Y], Z\right) T^{\uparrow}- \\
-2\left\{(d \sigma)\left(\left(\Pi_{H}[X, Y]\right)^{\uparrow}, Z^{\uparrow}\right)+A\left(\Pi_{H}[X, Y], Z\right)\right\} S+ \\
+\theta([X, Y])\left\{\left(\nabla_{T} Z\right)^{\uparrow}+(\phi Z)^{\uparrow}+4(d \sigma)\left(Z^{\uparrow}, T^{\uparrow}\right) S\right\}+\frac{1}{n+2} B(X, Y)(J Z)^{\uparrow} .
\end{gathered}
$$

Next (by $T\rfloor \Omega=T\rfloor A=0$ and the identities (34) and (36))

$$
\begin{gathered}
D_{\left[X^{\uparrow}, Y^{\uparrow}\right]} Z^{\uparrow}=\left(\nabla_{[X, Y]} Z\right)^{\uparrow}+ \\
+\Omega([X, Y], Z)\left\{T^{\uparrow}-\frac{\rho}{2(n+1)(n+2)} S\right\}-\frac{n+5}{n+2} A([X, Y], Z) S+ \\
+\frac{1}{n+2}\left\{\operatorname{Ric}_{\nabla}(X, J Y)(J Z)^{\uparrow}+\operatorname{Ric}_{\nabla}\left(\Pi_{H}[X, Y], J Z\right) S\right\}+ \\
+\frac{1}{n+2}\left\{(n-1) A(X, Y)-\frac{\rho}{2(n+1)} \Omega(X, Y)\right\}(J Z)^{\uparrow}+ \\
+\theta([X, Y])\left\{(\phi Z)^{\uparrow}+\frac{2}{n+2}\left[\varphi(Z)-\frac{1}{4(n+1)} Z(\rho)\right] S\right\} .
\end{gathered}
$$


Moreover (by (45)-(46))

(47)

$$
\begin{aligned}
& R^{D}\left(X^{\uparrow}, Y^{\uparrow}\right) Z^{\uparrow}=\left(\left[D_{X^{\uparrow}}, D_{Y^{\uparrow}}\right]-D_{\left[X^{\uparrow}, Y^{\uparrow}\right]}\right) Z^{\uparrow}=\left(\nabla_{X} \nabla_{Y} Z\right)^{\uparrow}+ \\
& +\left\{X(\Omega(Y, Z))+\Omega\left(X, \nabla_{Y} Z\right)\right\}\left\{T^{\uparrow}-\frac{\rho}{2(n+1)(n+2)} S\right\}- \\
& -\frac{X(\rho)}{2(n+1)(n+2)} \Omega(Y, Z) S-\frac{n+5}{n+2}\left\{X(A(Y, Z))+A\left(X, \nabla_{Y} Z\right)\right\} S+ \\
& +\frac{1}{n+2}\left\{X\left(\operatorname{Ric}_{\nabla}(Y, J Z)\right)+\operatorname{Ric}_{\nabla}\left(X, J \nabla_{Y} Z\right)\right\} S+ \\
& +\Omega(Y, Z)\left\{(\tau X)^{\uparrow}+(\phi X)^{\uparrow}-\frac{\rho}{4(n+1)(n+2)}(J X)^{\uparrow}\right\}+ \\
& +\frac{1}{2(n+2)}\left\{\operatorname{Ric}_{\nabla}(Y, J Z)-(n+5) A(Y, Z)\right\}(J X)^{\uparrow}-\left(\nabla_{Y} \nabla_{X} Z\right)^{\uparrow}- \\
& -\left\{Y(\Omega(X, Z))+\Omega\left(Y, \nabla_{X} Z\right)\right\}\left\{T^{\uparrow}-\frac{\rho}{2(n+1)(n+2)} S\right\}+ \\
& +\frac{Y(\rho)}{2(n+1)(n+2)} \Omega(X, Z) S+\frac{n+5}{n+2}\left\{Y(A(X, Z))+A\left(Y, \nabla_{X} Z\right)\right\} S- \\
& -\frac{1}{n+2}\left\{Y\left(\operatorname{Ric}_{\nabla}(X, J Z)\right)+\operatorname{Ric}_{\nabla}\left(Y, J \nabla_{X} Z\right)\right\} S- \\
& -\Omega(X, Z)\left\{(\tau Y)^{\uparrow}+(\phi Y)^{\uparrow}-\frac{\rho}{4(n+1)(n+2)}(J Y)^{\uparrow}\right\}- \\
& -\frac{1}{2(n+2)}\left\{\operatorname{Ric}_{\nabla}(X, J Z)-(n+5) A(X, Z)\right\}(J Y)^{\uparrow}-\left(\nabla_{[X, Y]} Z\right)^{\uparrow}- \\
& -\Omega([X, Y], Z)\left\{T^{\uparrow}-\frac{\rho}{2(n+1)(n+2)} S\right\}+\frac{n+5}{n+2} A([X, Y], Z) S- \\
& -\frac{1}{n+2}\left\{\operatorname{Ric}_{\nabla}(X, J Y)(J Z)^{\uparrow}+\operatorname{Ric}_{\nabla}\left(\Pi_{H}[X, Y], J Z\right) S\right\}- \\
& -\frac{1}{n+2}\left\{(n-1) A(X, Y)-\frac{\rho}{2(n+1)} \Omega(X, Y)\right\}(J Z)^{\uparrow}- \\
& -\theta([X, Y])\left\{(\phi Z)^{\uparrow}+\frac{2}{n+2}\left[\varphi(Z)-\frac{1}{4(n+1)} Z(\rho)\right] S\right\} .
\end{aligned}
$$

Using the identity

$$
[X, Y]=\nabla_{X} Y-\nabla_{Y} X+2 \Omega(X, Y) T, \quad X, Y \in H(M),
$$

one has

$$
X(\Omega(Y, Z))+\Omega\left(X, \nabla_{Y} X\right)-Y(\Omega(X, Z))-\Omega\left(Y, \nabla_{X} Z\right)-\Omega([X, Y], Z)=0
$$

as $\nabla \Omega=0$ and $T\rfloor \Omega=0$. Similarly (again by (47) and $T\rfloor A=0$ )

$$
-X(A(Y, Z))-A\left(X, \nabla_{Y} Z\right)+Y(A(X, Z))+A\left(Y, \nabla_{X} Z\right)+A([X, Y], Z)=
$$




$$
=-\left(\nabla_{X} A\right)(Y, Z)+\left(\nabla_{Y} A\right)(X, Z)
$$

Next (by $\nabla J=0)$

$$
\begin{gathered}
X\left(\operatorname{Ric}_{\nabla}(Y, J Z)\right)+\operatorname{Ric}_{\nabla}\left(X, J \nabla_{Y} Z\right)- \\
-Y\left(\operatorname{Ric}_{\nabla}(X, J Z)\right)-\operatorname{Ric}_{\nabla}\left(Y, J \nabla_{X} Z\right)-\operatorname{Ric}_{\nabla}\left(\Pi_{H}[X, Y], J Z\right)= \\
=\left(\nabla_{X} \operatorname{Ric}_{\nabla}\right)(Y, J Z)-\left(\nabla_{Y} \operatorname{Ric}_{\nabla}\right)(Y, J Z)+2 \Omega(X, Y) \operatorname{Ric}_{\nabla}(T, J Z) .
\end{gathered}
$$

Consequently (47) yields (38). The remaining identities (39)-(43) may be proved in a similar manner.

Using Lemma 2 one may compute the Ricci curvature of $\left(\mathfrak{M}, F_{\theta}\right)$. Let $\left\{E_{a}: 1 \leq a \leq 2 n\right\}$ be an orthonormal frame of $H(M)$ i.e. $G_{\theta}\left(E_{a}, E_{b}\right)=\delta_{a b}$. Then $\left\{\tilde{E}_{p}: 1 \leq p \leq 2 n+2\right\} \equiv\left\{E_{a}^{\uparrow}, T^{\uparrow} \pm S: 1 \leq a \leq 2 n\right\}$ with $\tilde{E}_{a}=E_{a}^{\uparrow}$, $\tilde{E}_{2 n+1}=T^{\uparrow}-S$ and $\tilde{E}_{2 n+2}=T^{\uparrow}+S$, is a local $F_{\theta}$-orthonormal frame of $T(\mathfrak{M})$, so that $\operatorname{Ric}_{D}(U, W)=\sum_{p=1}^{2 n+2} \epsilon_{p} F_{\theta}\left(R^{D}\left(\tilde{E}_{p}, W\right) U, \tilde{E}_{p}\right)$ i.e.

$$
\begin{gathered}
\operatorname{Ric}_{D}(U, W)=\sum_{a=1}^{2 n} F_{\theta}\left(R^{D}\left(E_{a}^{\uparrow}, W\right) U, E_{a}^{\uparrow}\right)+ \\
+2\left\{F_{\theta}\left(R^{D}\left(T^{\uparrow}, W\right) U, S\right)+F_{\theta}\left(R^{D}(S, W) U, T^{\uparrow}\right)\right\}
\end{gathered}
$$

for any $U, W \in \mathfrak{X}(\mathfrak{M})$. We may state the following

Lemma 3. For any $X, Y \in H(M)$

$$
\begin{aligned}
\operatorname{Ric}_{D}\left(X^{\uparrow}, Y^{\uparrow}\right) & =\frac{n+1}{n+2}\left\{\operatorname{Ric}_{\nabla}(X, Y)+3 A(X, J Y)\right\}+ \\
+ & \frac{\rho}{2(n+1)(n+2)} G_{\theta}(X, Y),
\end{aligned}
$$

$$
\begin{gathered}
\operatorname{Ric}_{D}\left(X^{\uparrow}, T^{\uparrow}\right)=\operatorname{Ric}_{\nabla}(X, T)+\operatorname{trace}\left\{\Pi_{H}(\nabla \phi) X\right\}+ \\
+\frac{1}{n+2} \varphi(J X)-2 \Omega(V, X)+\frac{1}{4(n+1)(n+2)} \Omega\left(X, \nabla^{H} \rho\right), \\
\operatorname{Ric}_{D}\left(X^{\uparrow}, S\right)=0, \\
\operatorname{Ric}_{D}\left(T^{\uparrow}, T^{\uparrow}\right)=\frac{1}{n+2} \operatorname{trace}\left\{\frac{\rho}{4(n+1)} J \phi-3(n+3) \tau^{2}\right\}+ \\
+\frac{1}{n+2} \operatorname{trace} G_{\theta} \Pi_{H}\left\{\operatorname{Ric}_{\nabla}(\cdot, J \phi \cdot)+\operatorname{Ric}_{\nabla}(\tau \cdot, J \cdot)-\right. \\
\left.-\nabla \varphi+\frac{1}{4(n+1)} \nabla d \rho+\frac{n+5}{2} \nabla_{T} A-\frac{1}{2}\left(\nabla_{T} \operatorname{Ric}_{\nabla}\right)(\cdot, J \cdot)\right\}, \\
\operatorname{Ric}_{D}\left(T^{\uparrow}, S\right)=\frac{\rho}{4(n+1)}, \quad \operatorname{Ric}_{D}(S, S)=\frac{n}{2} .
\end{gathered}
$$


Proof. Let $X, Y, E \in H(M)$ and let us replace $(X, Y, Z)$ in (38) by $(E, Y, X)$ and take the inner product of the resulting identity with $E^{\uparrow}$. As

$$
F_{\theta}\left(X^{\uparrow}, Y^{\uparrow}\right)=G_{\theta}(X, Y) \circ \pi, \quad F_{\theta}\left(X^{\uparrow}, T^{\uparrow}\right)=0, \quad F_{\theta}\left(X^{\uparrow}, S\right)=0,
$$

and $G_{\theta}(J X, J Y)=G_{\theta}(X, Y)$ we obtain

$$
\begin{gathered}
F_{\theta}\left(R^{D}\left(E^{\uparrow}, Y^{\uparrow}\right) X^{\uparrow}, E^{\uparrow}\right)=G_{\theta}\left(R^{\nabla}(E, Y) X, E\right)+ \\
+\Omega(Y, X)\left\{G_{\theta}(\tau E, E)+G_{\theta}(\phi E, E)\right\}- \\
-\Omega(E, X)\left\{G_{\theta}(\tau Y, E)+G_{\theta}(\phi Y, E)-\frac{\rho}{4(n+1)(n+2)} G_{\theta}(J Y, E)\right\}- \\
-\frac{1}{2(n+2)}\left\{\operatorname{Ric}_{\nabla}(E, J X)-(n+5) A(E, X)\right\} G_{\theta}(J Y, E)- \\
-\frac{1}{n+2} \operatorname{Ric}_{\nabla}(E, J Y) G_{\theta}(J X, E)- \\
-\frac{1}{n+2}\left\{(n-1) A(E, Y)-\frac{\rho}{2(n+1)} \Omega(E, Y)\right\} G_{\theta}(J X, E) .
\end{gathered}
$$

Let us replace $E$ by $E_{a}$ and sum over $1 \leq a \leq 2 n$. Since trace $(\tau)=0$ one obtains

$$
\sum_{a} F_{\theta}\left(R^{D}\left(E_{a}^{\uparrow}, Y^{\uparrow}\right) X^{\uparrow}, E_{a}^{\uparrow}\right)=\operatorname{Ric}_{\nabla}(X, Y)+
$$

$$
\begin{gathered}
+\Omega(Y, X) \operatorname{trace}(\phi)-\Omega(\tau Y, X)-\Omega(\phi Y, X)+\frac{\rho}{4(n+1)(n+2)} \Omega(J Y, X)- \\
-\frac{1}{2(n+2)}\left\{\operatorname{Ric}_{\nabla}(J Y, J X)-(n+5) A(J Y, X)\right\}-\frac{1}{n+2} \operatorname{Ric}_{\nabla}(J X, J Y)- \\
-\frac{1}{n+2}\left\{(n-1) A(J X, Y)-\frac{\rho}{2(n+1)} \Omega(J X, Y)\right\} .
\end{gathered}
$$

Note that (by the symmetry of $A$ together with $\tau \circ J+J \circ \tau=0$ )

$$
A(J X, Y)=A(X, J Y), \quad \Omega(\tau Y, X)=A(X, J Y) .
$$

To further simplify (55) we need some preparation. Let us replace $X$ by $J X$ in (33). One has

$$
\begin{gathered}
\operatorname{Ric}_{\nabla}(J X, J Y)=-2 i\left(R_{\mu \bar{v}} \theta^{\mu} \wedge \theta^{\bar{v}}\right)(J X, Y)-(n-1) A(J X, Y)= \\
=2 i\left(R_{\mu \bar{v}} \theta^{\mu} \wedge \theta^{\bar{v}}\right)(Y, J X)-(n-1) A(X, J Y)=
\end{gathered}
$$

(by applying (33) once again)

$$
=-\operatorname{Ric}_{\nabla}\left(Y, J^{2} X\right)-(n-1) A(Y, J X)-(n-1) A(X, J Y)
$$

or $\left(\right.$ as $J^{2}=-I$ on $\left.H(M)\right)$

$$
\operatorname{Ric}_{\nabla}(J X, J Y)=\operatorname{Ric}_{\nabla}(X, Y)-2(n-1) A(X, J Y)
$$


for any $X, Y \in H(M)$. Here we have also used the symmetry of $\operatorname{Ric}_{\nabla}$ on $H(M) \otimes H(M)$ i.e. $\operatorname{Ric}_{\nabla}(X, Y)=\operatorname{Ric}_{\nabla}(Y, X)$ which is an immediate consequence of (31)-(32). Moreover trace $(\phi)=0$ as a corollary of (29) and the fact that the trace of the endomorphism $\phi: H(M) \rightarrow H(M)$ coincides with the trace of its extension by $\mathbb{C}$-linearity to $H(M) \otimes \mathbb{C}$ (and $\phi_{\alpha}{ }^{\beta}$ is purely imaginary). Next one needs to compute $\Omega(\phi Y, X)$. If $\left\{T_{\alpha}: 1 \leq \alpha \leq n\right\}$ is a local frame of $T_{1,0}(M)$ and $X=X^{\alpha} T_{\alpha}+X^{\bar{\alpha}} T_{\bar{\alpha}}$ for some $X^{\alpha} \in C^{\infty}(U, \mathbb{C})$ (with $\overline{X^{\alpha}}=X^{\bar{\alpha}}$ ) then (by (29))

$$
\begin{gathered}
\Omega(\phi Y, X)= \\
=\frac{1}{2(n+2)}\left\{\operatorname{Ric}_{\nabla}\left(Y^{1,0}, X^{0,1}\right)+\operatorname{Ric}_{\nabla}\left(Y^{0,1}, X^{1,0}\right)\right\}- \\
-\frac{\rho}{4(n+1)(n+2)}\left\{G_{\theta}\left(Y^{1,0}, X^{0,1}\right)+G_{\theta}\left(Y^{0,1}, X^{1,0}\right)\right\}
\end{gathered}
$$

where we have set $X^{1,0}=X^{\alpha} T_{\alpha}$ and $X^{0,1}=\overline{X^{1,0}}$ (so that $X=X^{1,0}+X^{0,1}$ ). To further compute (57) let us observe that (by (32))

$$
\begin{gathered}
\operatorname{Ric}_{\nabla}\left(Y^{1,0}, X^{0,1}\right)+\operatorname{Ric}_{\nabla}\left(Y^{0,1}, X^{1,0}\right)= \\
=\operatorname{Ric}_{\nabla}(X, Y)-i(n-1)\left\{A\left(Y^{1,0}, X^{1,0}\right)-A\left(Y^{0,1}, X^{0,1}\right)\right\}=
\end{gathered}
$$

(as $A$ vanishes on $T_{1,0}(M) \otimes T_{0,1}(M)$, a consequence of $\tau T_{1,0}(M) \subset T_{0,1}(M)$ )

$$
=\operatorname{Ric}_{\nabla}(X, Y)-i(n-1)\left\{A\left(Y^{1,0}, X\right)-A\left(Y^{0,1}, X\right)\right\}
$$

or $\left(\right.$ as $\left.J Y=i\left(Y^{1,0}-Y^{0,1}\right)\right)$

(58) $\operatorname{Ric}_{\nabla}\left(Y^{1,0}, X^{0,1}\right)+\operatorname{Ric}_{\nabla}\left(Y^{0,1}, X^{1,0}\right)=\operatorname{Ric}_{\nabla}(X, Y)-(n-1) A(X, J Y)$.

Substitution from (58) into (57) leads to

$$
\begin{aligned}
\Omega(\phi Y, X)= & \frac{1}{2(n+2)}\left\{\operatorname{Ric}_{\nabla}(X, Y)-(n-1) A(X, J Y)\right\}- \\
& -\frac{\rho}{4(n+1)(n+2)} G_{\theta}(X, Y)
\end{aligned}
$$

for any $X, Y \in H(M)$. Substitution from (56) and (59) into (55) leads to

$$
\begin{gathered}
\sum_{a=1}^{2 n} F_{\theta}\left(R^{D}\left(E_{a}^{\uparrow}, Y^{\uparrow}\right) X^{\uparrow}, E_{a}^{\uparrow}\right)= \\
=\frac{n}{n+2} \operatorname{Ric}_{\nabla}(X, Y)+\frac{2(n-1)}{n+2} A(X, J Y)+\frac{\rho}{(n+1)(n+2)} G_{\theta}(X, Y) .
\end{gathered}
$$

Let us take the inner product of (39) with $S$ and use

$$
F_{\theta}(S, S)=0, \quad F_{\theta}\left(T^{\uparrow}, S\right)=\frac{1}{2}, \quad F_{\theta}\left(X^{\uparrow}, S\right)=0, \quad X \in H(M) .
$$


Since (by (39))

$$
R^{D}\left(X^{\uparrow}, T^{\uparrow}\right) Z^{\uparrow} \equiv\{\Omega(X, \phi Z)-\Omega(\tau X, Z)\} T^{\uparrow}, \quad \bmod H(M)^{\perp}, S,
$$

we obtain

$$
F_{\theta}\left(R^{D}\left(X^{\uparrow}, T^{\uparrow}\right) Z^{\uparrow}, S\right)=\frac{1}{2}\{\Omega(X, \phi Z)-\Omega(\tau X, Z)\} .
$$

Therefore the last two terms in (49) (with $U=X^{\uparrow}$ and $W=Y^{\uparrow}$ ) may be computed (by (61) and (59)) as

$$
\begin{gathered}
F_{\theta}\left(R^{D}\left(T^{\uparrow}, Y^{\uparrow}\right) X^{\uparrow}, S\right)+F_{\theta}\left(R^{D}\left(S, Y^{\uparrow}\right) X^{\uparrow}, T^{\uparrow}\right)= \\
=\frac{1}{2(n+2)}\left\{\operatorname{Ric}_{\nabla}(X, Y)+(n+5) A(X, J Y)\right\}-\frac{\rho}{4(n+1)(n+2)} G_{\theta}(X, Y) .
\end{gathered}
$$

Finally formulae (49) and (62) lead to (50). The remaining identities (51)(54) may be proved in a similar manner.

\section{Pseudohermitian Bochner-Lichnerowicz formula}

Let $f \in C^{\infty}(\mathfrak{M})$. Then $D f=\sum_{j=1}^{2 n+2} \epsilon_{j} \tilde{E}_{j}(f) \tilde{E}_{j}$ hence

$$
D(u \circ \pi)=\sum_{a} E_{a}(u) E_{a}^{\uparrow}+2 T(u) S=\left(\nabla^{H} u\right)^{\uparrow}+2 u_{0} S
$$

for any $u \in C^{\infty}(M)$, where $u_{0}=T(u)$. Next (by (50), (52) and (54))

$$
\operatorname{Ric}_{D}(D(u \circ \pi), D(u \circ \pi))=2 n u_{0}^{2}+
$$

$$
+\frac{n+1}{n+2}\left\{\operatorname{Ric}_{\nabla}\left(\nabla^{H} u, \nabla^{H} u\right)+3 A\left(\nabla^{H} u, J \nabla^{H} u\right)\right\}+\frac{\rho}{2(n+1)(n+2)}\left\|\nabla^{H} u\right\|^{2} .
$$

Let $u \in C^{\infty}(M)$ and $f=u \circ \pi \in C^{\infty}(\mathfrak{M})$. A straightforward calculation shows that

$$
\begin{gathered}
\left(D^{2} f\right)\left(X^{\uparrow}, Y^{\uparrow}\right)=\left(\nabla^{2} u\right)(X, Y)-\Omega(X, Y) u_{0}, \\
\left(D^{2} f\right)\left(X^{\uparrow}, T^{\uparrow}\right)=\left(\nabla^{2} u\right)(T, X)-(\phi X)(u), \\
\left(D^{2} f\right)\left(X^{\uparrow}, S\right)=-(1 / 2)(J X)(u), \\
\left(D^{2} f\right)\left(T^{\uparrow}, T^{\uparrow}\right)=T\left(u_{0}\right)-2 V(u), \\
\left(D^{2} f\right)\left(T^{\uparrow}, S\right)=0, \\
\left(D^{2} f\right)(S, S)=0,
\end{gathered}
$$

for every $X, Y \in H(M)$. Consequently

$$
\begin{gathered}
F_{\theta}^{*}\left(D^{2} f, D^{2} f\right)=\left\|\Pi_{H} \nabla^{2} u\right\|^{2}+2 n u_{0}^{2}-2 \operatorname{div}\left(J \nabla^{H} u\right)\left(u_{0}\right)+ \\
+4\left\{\left(J \nabla^{H} u\right)\left(u_{0}\right)-\left(\tau J \nabla^{H} u+\phi J \nabla^{H} u\right)(u)\right\} .
\end{gathered}
$$


By a result of J.M. Lee, [29], if $f=u \circ \pi$ then $\square f=\left(\Delta_{b} u\right) \circ \pi$ hence

$$
(D f)(\square f)=\left(\nabla^{H} u\right)\left(\Delta_{b} u\right), \quad F_{\theta}(D f, D f)=\left\|\nabla^{H} u\right\|^{2} .
$$

Finally (by taking into account the identities (64), (71) and (72) the BochnerLichnerowicz formula (21) becomes

$$
\begin{gathered}
-\frac{1}{2} \Delta_{b}\left(\left\|\nabla^{H} u\right\|^{2}\right)=\left\|\Pi_{H} \nabla^{2} u\right\|^{2}+4 n u_{0}^{2}-2 \operatorname{div}\left(J \nabla^{H} u\right) u_{0}+ \\
+4\left\{\left(J \nabla^{H} u\right)\left(u_{0}\right)-\left(\tau J \nabla^{H} u+\phi J \nabla^{H} u\right)(u)\right\}-\left(\nabla^{H} u\right)\left(\Delta_{b} u\right)+ \\
+\frac{n+1}{n+2}\left\{\operatorname{Ric}_{\nabla}\left(\nabla^{H} u, \nabla^{H} u\right)+3 A\left(\nabla^{H} u, J \nabla^{H} u\right)\right\}+\frac{\rho}{2(n+1)(n+2)}\left\|\nabla^{H} u\right\|^{2} .
\end{gathered}
$$

The term $\left(\phi J \nabla^{H} u\right)(u)$ may be expressed in terms of pseudohermitian Ricci curvature and torsion. As $J \nabla^{H} u=i\left(u^{\alpha} T_{\alpha}-u^{\bar{\alpha}} T_{\bar{\alpha}}\right)$ with $u^{\alpha}=g^{\alpha \bar{\beta}} u_{\bar{\beta}}$ and $u_{\bar{\beta}}=T_{\bar{\beta}}(u)$ one has (by (29))

$$
\begin{gathered}
\phi J \nabla^{H} u=i\left(u^{\alpha} \phi_{\alpha}{ }^{\beta} T_{\beta}-u^{\bar{\alpha}} \phi_{\bar{\alpha}}^{\bar{\beta}} T_{\bar{\beta}}\right)= \\
=-\frac{1}{2(n+2)}\left\{g^{\beta \bar{v}} \operatorname{Ric}_{\nabla}\left(\left(\nabla^{H} u\right)^{1,0}, T_{\bar{v}}\right) T_{\beta}+\right. \\
\left.+g^{\bar{\beta} v} \operatorname{Ric}_{\nabla}\left(\left(\nabla^{H} u\right)^{0,1}, T_{v}\right) T_{\bar{\beta}}\right\}+\frac{\rho}{4(n+1)(n+2)} \nabla^{H} u
\end{gathered}
$$

hence (as $\operatorname{Ric}_{\nabla}$ is symmetric on $H(M) \otimes H(M)$ )

$$
\begin{gathered}
\left(\phi J \nabla^{H} u\right)(u)=\frac{\rho}{4(n+1)(n+2)}\left\|\nabla^{H} u\right\|^{2}- \\
-\frac{1}{n+2} \operatorname{Ric}_{\nabla}\left(\left(\nabla^{H} u\right)^{1,0},\left(\nabla^{H} u\right)^{0,1}\right) .
\end{gathered}
$$

Formula (32) implies

$$
\operatorname{Ric}_{\nabla}\left(X^{1,0}, X^{0,1}\right)=\frac{1}{2}\left\{\operatorname{Ric}_{\nabla}(X, X)-(n-1) A(X, J X)\right\}
$$

for any $X \in H(M)$. Hence (by (75) with $X=\nabla^{H} u$ ) formula (74) becomes

$$
\begin{gathered}
\left(\phi J \nabla^{H} u\right)(u)=\frac{\rho}{4(n+1)(n+2)}\left\|\nabla^{H} u\right\|^{2}- \\
-\frac{1}{2(n+2)}\left\{\operatorname{Ric}_{\nabla}\left(\nabla^{H} u, \nabla^{H} u\right)-(n-1) A\left(\nabla^{H} u, J \nabla^{H} u\right)\right\} .
\end{gathered}
$$

Let us substitute from (76) and $\left(\tau J \nabla^{H} u\right)(u)=A\left(\nabla^{H} u, J \nabla^{H} u\right)$ into (73). We obtain

$$
\begin{gathered}
-\frac{1}{2} \Delta_{b}\left(\left\|\nabla^{H} u\right\|^{2}\right)=\left\|\Pi_{H} \nabla^{2} u\right\|^{2}-\left(\nabla^{H} u\right)\left(\Delta_{b} u\right)+4 n u_{0}^{2}+ \\
+4\left(J \nabla^{H} u\right)\left(u_{0}\right)-2 \operatorname{div}\left(J \nabla^{H} u\right) u_{0}+\frac{n+3}{n+2} \operatorname{Ric}_{\nabla}\left(\nabla^{H} u, \nabla^{H} u\right)-
\end{gathered}
$$




$$
-\frac{\rho}{2(n+1)(n+2)}\left\|\nabla^{H} u\right\|^{2}-\frac{3(n+1)}{n+2} A\left(\nabla^{H} u, J \nabla^{H} u\right) .
$$

A straightforward calculation shows that for any $u \in C^{\infty}(M)$

$$
\operatorname{div}\left(J \nabla^{H} u\right)=2 n u_{0} .
$$

By (78) identity (77) simplifies to

$$
\begin{gathered}
-\frac{1}{2} \Delta_{b}\left(\left\|\nabla^{H} u\right\|^{2}\right)=\left\|\Pi_{H} \nabla^{2} u\right\|^{2}-\left(\nabla^{H} u\right)\left(\Delta_{b} u\right)+ \\
+4\left(J \nabla^{H} u\right)\left(u_{0}\right)+\frac{n+3}{n+2} \operatorname{Ric}_{\nabla}\left(\nabla^{H} u, \nabla^{H} u\right)- \\
-\frac{\rho}{2(n+1)(n+2)}\left\|\nabla^{H} u\right\|^{2}-\frac{3(n+1)}{n+2} A\left(\nabla^{H} u, J \nabla^{H} u\right) .
\end{gathered}
$$

(the pseudohermitian Bochner-Lichnerowicz formula). Let us integrate over $M$ and observe that (by Green's lemma and (78))

$$
\int_{M}\left(J \nabla^{H} u\right)\left(u_{0}\right) \Psi_{\theta}=-\int_{M} u_{0} \operatorname{div}\left(J \nabla^{H} u\right) \Psi_{\theta}=-2 n\left\|u_{0}\right\|_{L^{2}}^{2} .
$$

We obtain

$$
\begin{gathered}
\left\|\Pi_{H} \nabla^{2} u\right\|_{L^{2}}^{2}-8 n\left\|u_{0}\right\|_{L^{2}}^{2}+ \\
+\int_{M}\left\{\frac{n+3}{n+2} \operatorname{Ric}_{\nabla}\left(\nabla^{H} u, \nabla^{H} u\right)-\frac{3(n+1)}{n+2} A\left(\nabla^{H} u, J \nabla^{H} u\right)\right\} \Psi_{\theta}= \\
=\int_{M}\left(\nabla^{H} u\right)\left(\Delta_{b} u\right) \Psi_{\theta}+\frac{1}{2(n+1)(n+2)} \int_{M} \rho\left\|\nabla^{H} u\right\|^{2} \Psi_{\theta}
\end{gathered}
$$

(the integral pseudohermitian Bochner-Lichnerowicz formula).

6. A LOWER BOUND ON $\lambda_{1}(\theta)$

Let $\lambda \in \sigma\left(\Delta_{b}\right)$ be an eigenvalue of $\Delta_{b}$ and $u \in \operatorname{Eigen}\left(\Delta_{b}, \lambda\right)$ an eigenfunction corresponding to $\lambda$. With these data

$$
\int_{M}\left(\nabla^{H} u\right)\left(\Delta_{b} u\right) \Psi_{\theta}=\lambda\left\|\nabla^{H} u\right\|_{L^{2}}^{2} .
$$

On the other hand (cf. (27) in [4], p. 88)

$$
\left\|\Pi_{H} \nabla^{2} u\right\|^{2} \geq \frac{1}{2 n}\left(\Delta_{b} u\right)^{2}
$$

everywhere on $M$. Moreover (by Green's lemma)

$$
\left\|\Delta_{b} u\right\|_{L^{2}}^{2}=\lambda \int_{M} u \Delta_{b} u \Psi_{\theta}=\lambda\left\|\nabla^{H} u\right\|_{L^{2}}^{2} .
$$

By our assumption (5)

$$
\int_{M} \operatorname{Ric}_{\nabla}\left(\nabla^{H} u, \nabla^{H} u\right) \Psi_{\theta} \geq k\left\|\nabla^{H} u\right\|_{L^{2}}^{2} .
$$


Moreover (by (5) with $X=E_{a}$ )

$$
\rho \geq n k \text {. }
$$

In particular $\rho_{0} \equiv \sup _{x \in M} \rho(x)>0$ and

$$
\int_{M} \rho\left\|\nabla^{H} u\right\|^{2} \Psi_{\theta} \leq \rho_{0}\left\|\nabla^{H} u\right\|_{L^{2}}^{2} .
$$

For any $X, Y \in H(M)$ (by Cauchy-Schwartz inequality)

$$
\begin{gathered}
|A(X, Y)|=\left|G_{\theta}(X, \tau Y)\right| \leq\|X\|\|\tau Y\| \leq\|\tau\|\|X\|\|Y\|, \\
\|\tau\|_{x}=\sup \left\{G_{\theta, x}\left(\tau_{x} v, \tau_{x} v\right): v \in H(M)_{x}, \quad G_{\theta, x}(v, v)=1\right\}, \quad x \in M .
\end{gathered}
$$

Consequently

$$
\int_{M} A\left(\nabla^{H} u, J \nabla^{H} u\right) \leq \tau_{0}\left\|\nabla^{H} u\right\|_{L^{2}}^{2}
$$

where $\tau_{0}=\sup _{x \in M}\|\tau\|_{x}$. The integral Bochner-Lichnerowicz formula (80) reads (by (81))

$$
\begin{gathered}
0=\left\|\Pi_{H} \nabla^{2} u\right\|_{L^{2}}^{2}-8 n\left\|u_{0}\right\|_{L^{2}}^{2}+ \\
+\int_{M}\left\{\frac{n+3}{n+2} \operatorname{Ric}_{\nabla}\left(\nabla^{H} u, \nabla^{H} u\right)-\frac{3(n+1)}{n+2} A\left(\nabla^{H} u, J \nabla^{H} u\right)\right\} \Psi_{\theta}- \\
-\lambda\left\|\nabla^{H} u\right\|_{L^{2}}^{2}-\frac{1}{2(n+1)(n+2)} \int_{M} \rho\left\|\nabla^{H} u\right\|^{2} \Psi_{\theta} \geq
\end{gathered}
$$

(by (82) and (84)-(87))

$$
\begin{gathered}
\geq \frac{1}{2 n}\left\|\Delta_{b} u\right\|_{L^{2}}^{2}-8 n\left\|u_{0}\right\|_{L^{2}}^{2}+\left[\frac{(n+3) k}{n+2}-\frac{3(n+1) \tau_{0}}{n+2}\right]\left\|\nabla^{H} u\right\|_{L^{2}}^{2}- \\
-\lambda\left\|\nabla^{H} u\right\|_{L^{2}}^{2}-\frac{\rho_{0}}{2(n+1)(n+2)}\left\|\nabla^{H} u\right\|_{L^{2}}^{2}
\end{gathered}
$$

so that (by (83))

$$
\begin{gathered}
\left\{\frac{1}{2 n}-1+\frac{1}{\lambda}\left[\frac{(n+3) k}{n+2}-\frac{3(n+1) \tau_{0}}{n+2}-\right.\right. \\
\left.\left.-\frac{\rho_{0}}{2(n+1)(n+2)}\right]\right\}\left\|\Delta_{b} u\right\|_{L^{2}}^{2} \leq 8 n\left\|u_{0}\right\|_{L^{2}}^{2} .
\end{gathered}
$$

Finally (by (83) and Chang-Chiu inequality (91) in Appendix A)

$$
-\frac{2 n+3}{n+2}+\frac{1}{\lambda}\left\{\frac{(n+3) k}{2(n+1)}-\frac{(11 n+19) \tau_{0}}{n+2}-\frac{\rho_{0}}{2(n+1)(n+2)}\right\} \leq 0
$$

or

$$
\lambda \geq \frac{2 n}{(n+2)(n+3)}\left\{(n+3) k-(11 n+19) \tau_{0}-\frac{\rho_{0}}{2(n+1)}\right\}
$$


which is the announced lower bound on $\lambda_{1}(\theta)$ (cf. (6) above). Of course this is useful only when

$$
k>\frac{(11 n+19) \tau_{0}}{n+3}+\frac{\rho_{0}}{2(n+1)(n+3)} .
$$

In particular (by (85)) it must be $k>2(n+1)(11 n+19) \tau_{0} /[(n+2)(2 n+3)]$. To parallel the estimate (6) in Theorem 1 to A. Greenleaf's estimate (7) let $g_{\theta}$ be a Sasakian metric. If this is the case the assumption

$$
R_{\lambda \bar{\mu}} Z^{\lambda} Z^{\bar{\mu}}+\frac{i n}{2}\left(A_{\bar{\alpha} \bar{\beta}} Z^{\bar{\alpha}} Z^{\bar{\beta}}-A_{\alpha \beta} Z^{\alpha} Z^{\beta}\right) \geq k g_{\lambda \bar{\mu}} Z^{\lambda} Z^{\bar{\mu}}
$$

(with $Z^{\bar{\alpha}}=\overline{Z^{\alpha}}$ ) in [23], p. 192, is equivalent to (5). Also (6) becomes

$$
\lambda_{1}(\theta) \geq \frac{2 n}{(n+2)(n+3)}\left\{(n+3) k-\frac{\rho_{0}}{2(n+1)}\right\}
$$

and right hand side of (90) is larger (closer to $\lambda_{1}(\theta)$ from below) than right hand side of (7) precisely when (8) holds. In particular if $M=S^{2 n+1}$ then the Tanaka-Webster connection of the canonical contact form $\theta=$ $(i / 2)(\bar{\partial}-\partial)|z|^{2}$ has curvature (cf. [12])

$R^{\nabla}(X, Y) Z=g_{\theta}(Y, Z) X-g_{\theta}(X, Z) Y+\Omega(X, Z) J Y-\Omega(Y, Z) J X+2 \Omega(X, Y) J Z$

for any $X, Y, Z \in H\left(S^{2 n+1}\right)$. Consequently the pseudohermitian Ricci and scalar curvature of the sphere are $R_{\lambda \bar{\mu}}=2(n+1) g_{\lambda \bar{\mu}}$ and $\rho=2 n(n+1)$ so that (8) becomes $k>2(n+1) /(n+3)$ (which is clearly satisfied by $k=2(n+1))$.

\section{Appendix A. The Chang-Chiu inequality}

The purpose of Appendix A is to give a proof of

$$
4 n\left\|u_{0}\right\|_{L^{2}}^{2} \leq \frac{1}{n}\left\|\Delta_{b} u\right\|_{L^{2}}^{2}+4 \tau_{0}\left\|\nabla^{H} u\right\|_{L^{2}}^{2}
$$

for any $u \in C^{\infty}(M, \mathbb{R})$ (compare ${ }^{2}$ to (3.5) in [10], p. 270). This is referred to as the Chang-Chiu inequality. To prove (91) let us contract (13) by $u^{\beta}$ so that to obtain $u^{\beta} \nabla_{0} u_{\beta}=u^{\beta} \nabla_{\beta} u_{0}-A_{\alpha \beta} u^{\alpha} u^{\beta}$ or

$$
u^{\beta} \nabla_{0} u_{\beta}=\nabla_{\beta}\left(u_{0} u^{\beta}\right)-u_{0} \nabla_{\beta} u^{\beta}-A_{\alpha \beta} u^{\alpha} u^{\beta} .
$$

\footnotetext{
${ }^{2}$ Discrepancies among (91) and (3.5) in [10], p. 270, are due to the different convention as to wedge products of 1 -forms producing the additional 2 factor in (12). Cf. also (1.62) in [12], p. 39, and (9.7) in [12], p. 424. Through this paper conventions as to wedge products and exterior differentiation calculus are those in [28], p. 35-36.
} 
On the other hand (by (12)) $\nabla_{\beta} u^{\beta}=\nabla_{\bar{\beta}} u^{\bar{\beta}}-2 i n u_{0}$ so that (by substitution into (92))

$$
u^{\beta} \nabla_{0} u_{\beta}+u_{0} \nabla_{\bar{\beta}} u^{\bar{\beta}}=2 \operatorname{in} u_{0}^{2}-A_{\alpha \beta} u^{\alpha} u^{\beta}+\nabla_{\beta}\left(u_{0} u^{\beta}\right) .
$$

Next (again by (13)) $u_{0} \nabla_{\bar{\beta}} u^{\bar{\beta}}=\nabla_{\bar{\beta}}\left(u_{0} u^{\bar{\beta}}\right)-u^{\bar{\beta}}\left(\nabla_{0} u_{\bar{\beta}}+u_{\gamma} A_{\bar{\beta}}^{\gamma}\right)$ hence (by substitution of $u_{0} \nabla_{\bar{\beta}} u^{\bar{\beta}}$ into (93))

$$
\begin{gathered}
i\left(u^{\bar{\beta}} \nabla_{0} u_{\bar{\beta}}-u^{\beta} \nabla_{0} u_{\beta}\right)= \\
=2 n u_{0}^{2}+i\left(A_{\alpha \beta} u^{\alpha} u^{\beta}-A_{\bar{\alpha} \bar{\beta}} u^{\bar{\alpha}} u^{\bar{\beta}}\right)+i\left\{\nabla_{\bar{\alpha}}\left(u_{0} u^{\bar{\alpha}}\right)-\nabla_{\alpha}\left(u_{0} u^{\alpha}\right)\right\}
\end{gathered}
$$

(compare to (2.4) in Lemma 2.2, [10], p. 268). Calculations are performed with respect to an arbitrary local frame $\left\{T_{\alpha}: 1 \leq \alpha \leq n\right\}$ in $T_{1,0}(M)$ (rather than a $G_{\theta}$-orthonormal frame, as in [10]). The next step is to evaluate the left hand side of (94) in terms of the operator $P+\bar{P}$. One has $u_{0}=(i / 2 n)\left(\nabla_{\beta} u^{\beta}-\nabla_{\bar{\beta}} u^{\bar{\beta}}\right)$ hence (by (13))

$$
u^{\bar{\alpha}} \nabla_{0} u_{\bar{\alpha}}=\frac{i}{2 n} u^{\bar{\alpha}}\left(u_{\bar{\alpha}}^{\bar{\gamma}} \bar{\gamma}-u_{\bar{\alpha}}^{\gamma} \gamma\right)-A_{\bar{\alpha} \bar{\beta}} u^{\bar{\alpha}} u^{\bar{\beta}} .
$$

Using $P_{\bar{\alpha}} u \equiv u_{\bar{\alpha}^{\gamma}}^{\gamma}{ }_{\gamma}-2 n i A_{\bar{\alpha} \bar{\beta}} u^{\bar{\beta}}$ the identity (95) becomes

$$
i u^{\bar{\alpha}} \nabla_{0} u_{\bar{\alpha}}=\frac{1}{2 n} u^{\bar{\alpha}}\left(P_{\bar{\alpha}} u-u_{\bar{\alpha}}^{\bar{\gamma}} \bar{\gamma}\right) .
$$

Let us take the complex conjugate of (96) and add the resulting equation to (96). We obtain

$$
2 n i\left(u^{\bar{\alpha}} \nabla_{0} u_{\bar{\alpha}}-u^{\beta} \nabla_{0} u_{\beta}\right)=u^{\bar{\alpha}} P_{\bar{\alpha}} u+u^{\alpha} P_{\alpha} u-\left\{u^{\bar{\alpha}} u_{\bar{\alpha}}^{\bar{\gamma}} \bar{\gamma}+u^{\alpha} u_{\alpha}^{\gamma} \gamma\right\}
$$

where $P_{\alpha} u \equiv u_{\alpha} \bar{\gamma}_{\bar{\gamma}}+2 n i A_{\alpha \beta} u^{\beta}$. Let us replace $u^{\alpha} u_{\alpha}^{\beta}{ }_{\beta}+u^{\bar{\alpha}} u_{\alpha^{\alpha}} \bar{\beta}_{\bar{\beta}}$ from (18) into (97). We obtain

$$
\begin{gathered}
2 n i\left(u^{\bar{\alpha}} \nabla_{0} u_{\bar{\alpha}}-u^{\alpha} \nabla_{0} u_{\alpha}\right)=2\left(u^{\alpha} P_{\alpha} u+u^{\bar{\alpha}} P_{\bar{\alpha}} u\right)- \\
-2 n i\left(A_{\alpha \beta} u^{\alpha} u^{\beta}-A_{\bar{\alpha} \bar{\beta}} u^{\bar{\alpha}} u^{\bar{\beta}}\right)+\left(\nabla^{H} u\right)\left(\Delta_{b} u\right) .
\end{gathered}
$$

Finally substitution from (98) into (94) leads to

$$
\begin{gathered}
2\left(u^{\alpha} P_{\alpha}+u^{\bar{\alpha}} P_{\bar{\alpha}} u\right)+\left(\nabla^{H} u\right)\left(\Delta_{b} u\right)= \\
=4 n^{2} u_{0}^{2}+4 n i\left(A_{\alpha \beta} u^{\alpha} u^{\beta}-A_{\bar{\alpha} \bar{\beta}} u^{\bar{\alpha} \bar{\beta}}\right)+2 n i\left\{\nabla_{\bar{\alpha}}\left(u_{0} u^{\bar{\alpha}}\right)-\nabla_{\alpha}\left(u_{0} u^{\alpha}\right)\right\} .
\end{gathered}
$$

Let us observe that

$$
\begin{gathered}
i\left(A_{\alpha \beta} u^{\alpha} u^{\beta}-A_{\bar{\alpha} \bar{\beta}} u^{\bar{\alpha} \bar{\beta}}\right)=A\left(\nabla^{H} u, J \nabla^{H} u\right), \\
i\left\{\nabla_{\alpha}\left(u_{0} u^{\alpha}\right)-\nabla_{\bar{\alpha}}\left(u_{0} u^{\bar{\alpha}}\right)\right\}=\operatorname{div}\left(u_{0} J \nabla^{H} u\right),
\end{gathered}
$$


and $u^{\alpha} P_{\alpha}+u^{\bar{\alpha}} P_{\bar{\alpha}} u=g_{\theta}^{*}\left(L u, d_{b} u\right)$ where $L=P+\bar{P}$. Then (99) becomes

$$
\begin{aligned}
& 2 g_{\theta}^{*}\left(L u, d_{b} u\right)+\left(\nabla^{H} u\right)\left(\Delta_{b} u\right)=4 n^{2} u_{0}^{2}+ \\
& +4 n A\left(\nabla^{H} u, J \nabla^{H} u\right)-2 n \operatorname{div}\left(u_{0} J \nabla^{H} u\right) .
\end{aligned}
$$

Let us integrate over $M$ and use Green's lemma. Then (by Lemma 1)

$$
\begin{aligned}
& -2 \int_{M}\left(P_{0} u\right) u \Psi_{\theta}+\int_{M}\left(\nabla^{H} u\right)\left(\Delta_{b} u\right) \Psi_{\theta}= \\
& =4 n^{2}\left\|u_{0}\right\|_{L^{2}}^{2}+4 n \int_{M} A\left(\nabla^{H} u, J \nabla^{H} u\right) \Psi_{\theta} .
\end{aligned}
$$

Also (again by Green's lemma)

$$
\int_{M}\left(\nabla^{H} u\right)\left(\Delta_{b} u\right) \Psi_{\theta}=\int_{M}\left(\Delta_{b} u\right)^{2} \Psi_{\theta}=\left\|\Delta_{b} u\right\|_{L^{2}}^{2} .
$$

Finally as $P_{0}$ is nonnegative (87) and (101) lead to (91). Q.e.d.

\section{REFERENCES}

[1] A. Aribi \& A. El Soufi, Inequalities and bounds for the eigenvalues of the sub-Laplacian on a strictly pseudoconvex CR manifold, Calculus of Variations and Partial Differential Equations, (17 May 2012), pp. 1-27, doi : $10.1007 / \mathrm{s} 00526-012-0523-2$

[2] A. Aribi \& S. Dragomir \& A. El Soufi, On the continuity of the eigenvalues of a sublaplacian, Canadian Mathematical Bulletin, http://dx.doi.org/10.4153/CMB-2012-026-9, 13 pages, Published: 2012-09-21.

[3] E. Barletta \& S. Dragomir, On the spectrum of a strictly pseudoconvex CR manifold, Abhandlungen Math. Sem. Univ. Hamburg, 67(1997), 143-153.

[4] E. Barletta, The Lichnerowicz theorem on CR manifolds, Tsukuba J. Math., (1)31(2007), 77-97.

[5] E. Barletta \& S. Dragomir \& K.L. Duggal, Foliations in Cauchy-Riemann geometry, Mathematical Surveys and Monographs, Vol. 140, American Mathematical Society, 2007.

[6] E. Barletta \& S. Dragomir \& H. Urakawa, Pseudoharmonic maps from a nondegenerate CR manifold into a Riemannian manifold, Indiana University Math. J., (2)50(2001), 719-746; Yang-Mills fields on CR manifolds, J. Math. Phys., (8)47(2008), 083504 1-41.

[7] F. Baudoin \& N. Garofalo, Generalized Bochnerformulas and Ricci lower bounds for sub-Riemannian manifolds of rank two, preprint, arXiv:0904.1623v1 [math.DG] 10 Apr 2009

[8] M. Berger \& P. Gauduchon \& E. Mazet, Le spectre d'une variété Riemannienne, Lecture Notes in Math., 194, Springer-Verlag, Berlin-New York, 1971.

[9] J-M. Bony, Principe du maximum, inégalite de Harnack et unicité du probleme de Cauchy pour les opérateurs elliptiques dégénérés, Ann. Inst. Fourier (Grenoble), (1)19(1969), $277 \mathrm{D} 304$. 
[10] S-C. Chang \& H-L. Chiu, Nonnegativity of CR Paneitz operator and its application to the CR Obata's theorem, J. Geom. Anal., 19(2009), 261-287, DOI $10.1007 / \mathrm{s} 12220-008-9060-9$

[11] H-L. Chiu, The sharp lower bound for the first positive eigenvalue of the sublaplacian on a pseudohermitian 3-manifold, Ann. Global Analysis and Geometry, 30(2006), 81-96.

[12] S. Dragomir \& G. Tomassini, Differential Geometry and Analysis on CR Manifolds, Progress in Mathematics, Vol. 246, Birkhäuser, Boston-Basel-Berlin, 2006.

[13] A. El Soufi \& S. Ilias, Immersions minimales, premiere valeur propre du Laplacien et volume conforme, Mathematische Annalen, 275(1986), 257-267; Une inegalité du type "Reilly" pour les sous-variétés de l'espace hyperbolique, Commentarii Mathematici Helvetici, 67(1992), 167-181; Majoration de la seconde valeur propre d'un operateur de Schrodinger sur une variété compacte et applications, Journal of Functional Analysis, (2)103(1992), 294-316; Riemannian manifolds admitting isometric immersions by their $1^{\text {rst }}$ eigenfunctions, Pacific Journal of Mathematics, (1)195(2000), 91-99; Second eigenvalue of Schrodinger operators and mean curvature of a compact submanifold, Communications in Mathematical Physics, 208(2000), 761-770; Extremal metrics for the $1^{\text {rst }}$ eigenvalue of the Laplacian in a conformal class, Proceedings of the American Mathematical Society, (5)131(2003), 1611-1618; Domain deformations and eigenvalues of the Dirichlet Laplacian in a Riemannian manifold, Illinois Journal of Mathematics, 51(2007), 645-666; Laplacian eigenvalues functionals and metric deformations on compact manifolds, Journal of Geometry and Physics, (1)58(2008), 89-104.

[14] A. El Soufi \& S. Ilias \& A. Ros, Sur la Premiere valeur propre des Tores, Seminaire de Theorie Spectrale et Geometrie de l'Institut Fourier, Vol. 15, Annee 1996-97, pp. 17-23.

[15] A. El Soufi \& B. Colbois, Extremal eigenvalues of the Laplacian in a conformal class of metrics: the "conformal spectrum", Annals of Global Analysis and Geometry, (4)24(2003), 337-349; Eigenvalues of the Laplacian acting on p-forms and metric conformal deformations, Proceedings of the American Mathematical Society, (3)134(2006), 715-721.

[16] A. El Soufi \& B. Colbois \& E. Dryden, G-invariant eigenvalues of G-invariant metrics on compact manifolds, Mathematische Zeitschrift, 258(2007), 29-41.

[17] A. El Soufi \& N. Moukadem, Critical Potentials for the Eigenvalues of Schrodinger Operators, Journal of Mathematical Analysis and Applications, 314(2006), 195-209.

[18] A. El Soufi \& H. Giacomini \& M. Jazar, A unique extremal metric for the least eigenvalue of the Laplacian on the Klein bottle, Duke Mathematical Journal, (1)135(2006), 181-202.

[19] A. El Soufi \& R. Kiwan, Extremal $1^{\text {rst }}$ Dirichlet eigenvalue of doubly connected plane domains and dihedral symmetry, SIAM Journal on Mathematical Analysis, (4)39(2007), 1112-1119; Extremal property of spherical shells with respect to the second Dirichlet eigenvalue, Communications on Pure and Applied Analysis, (5)7(2008), 1193-1201.

[20] C. Fefferman, Monge-Ampere equations, the Bergman kernel, and geometry of pseudoconvex domains, Ann. of Math., (2)103(1976), 395-416; 104(1976), 393394. 
[21] C.R. Graham, On Sparling's characterization of Fefferman metrics, American J. Math., 109(1987), 853-874.

[22] C.R. Graham \& J.M. Lee, Smooth solutions of degenerate Laplacians on strictly pseudoconvex domains, Duke Math. J., 57(1988), 697-720.

[23] A. Greenleaf, The first eigenvalue of a sublaplacian on a pseudohermitian manifold, Commun. Partial Differential Equations, (2)10(1985), 191-217.

[24] S. Ivanov \& D. Vassilev, An Obata type result for the first eigenvalue of the sub-laplacian on a CR manifold with a divergence free torsion, preprint, arXiv: 1203.5812v1 [math.DG] 26 Mar 2012

[25] D. Jerison \& J.M. Lee, The Yamabe problem on CR manifolds, J. Diff. Geometry, 25(1987), 167-197.

[26] J. Jost \& C-J. Xu, Subelliptic harmonic maps, Trans. A.M.S., (11)350(1998), 4633-4649.

[27] S-D. Jung \& K-R. Lee \& K. Richardson, Generalized Obata theorem and its applications on foliations, arXiv:0908.4545v1 [math.DG] 31 Aug 2009

[28] S. Kobayashi \& K. Nomizu, Foundations of differential geometry, Interscience Publishers, New York, Vol. I, 1963, Vol. II, 1969.

[29] J.M. Lee, The Fefferman metric and pseudohermitian invariants, Trans. A.M.S., (1)296(1986), 411-429.

[30] J.M. Lee, Pseudo-Einstein structures on CR manifolds, Amer. J. Math., 110(1988), 157-178.

[31] J. Lee \& K. Richardson, Lichnerowicz and Obata theorems for foliations, Pacific J. Math., (2)206(2002), 339-357.

[32] S-Y. Li \& H-S. Luk, The sharp lower bound for the first positive eigenvalue of a sub-Laplacian on a pseudohermitian manifold, Proc. Amer. Math. Soc., (3)132(2004), 789-798.

[33] A. Menikoff \& J. Sjöstrand, On the eigenvalues of a class of hypoelliptic operators, Math. Ann., 235(1978), 55-58.

[34] M. Obata, Certain conditions for a Riemannian manifold to be isometric with a sphere, J. Math. Soc. Japan, 14(1962), 333-340.

[35] B. O'Neill, The fundamental equations of a submersion, Michigan Math. J., 13(1966), 459-469.

[36] H-K. Pak \& J-H. Park, A note on generalized Lichnerowicz-Obata theorem for Riemannian foliations, Bull. Korean Math. Soc., (4)48(2011), 769-777. D0I 10.4134/BKMS . 2011.48.4.769

[37] N. Tanaka, A differential geometric study on strongly pseudo-convex manifolds, Kinokuniya Book Store Co., Ltd., Kyoto, 1975.

[38] S.M. Webster, Pseudohermitian structures on a real hypersurface, J. Differential Geometry, 13(1978), 25-41. 